\title{
OPERATOR DYNAMICS IN MOLECULAR BIOLOGY
}

\author{
Tsuyoshi KATO
}

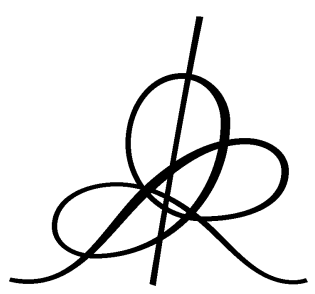

Institut des Hautes Études Scientifiques 35 , route de Chartres 91440 - Bures-sur-Yvette (France)

Août 2001

IHES/M/01/41 


\section{OPERATOR DYNAMICS IN MOLECULAR BIOLOGY}

\section{T.KATO}

\section{Introduction}

Recent develpment of molecular biology revealed some mathematical nature of the structure of genes. Basic elements of genes are DNA, which are (double helixed) sequences consisted by four bases, $X=\{A, T, G, C\}$. A primary structure of genes is just a finite sequence of $X$. There are higher structures, at least until fourth ones. These are related to folding of proteins, which reflects some phisical force.

One way to represent molecular biology is a mathematical formulation of molecular activity of life. Here we will use algebra $A$ as a box which contains all informations in the formulation. Multiplication of elements of $A$ corresponds to composition of functions of genes. Here one will use involutive algebras, in particular $C^{*}$ ones, and will represent phisical states by vectors in $A$.

Frist we will fix a set $X=\left\{X_{1}, \ldots, X_{n}, \ldots\right\} \subset A$, whose elements will consist of primary structure. A primary structure is just a sequence of finite length by elements of $X$ :

$$
V=v_{1} v_{2} \ldots v_{l}, \quad v_{i} \in X .
$$

Then we will express higher structure of $V$ by elements $a_{1}, \ldots, a_{l+1} \in A$ where we will force shift relation as:

$$
a_{i} a_{i+1}^{*}=v_{i}, \quad i=1,2, \ldots, l .
$$

Combining these, we will express total structure as:

$$
V=a_{1}\left[v_{1}\right] a_{2}\left[v_{2}\right] \ldots a_{l}\left[v_{l}\right] a_{l+1} .
$$

We will call this as a $m$-dna sequence. The sequence $\left(a_{1}, \ldots, a_{l+1}\right)$ will be called as a state set which should represent higher structures. State sets will be more flexible than the primary sets. For example folding of proteins can change by 
phisical force, like catalyzing as ensymes. The energy of the state at $v_{i}$ is defined as:

$$
\text { trace } a_{i} a_{i}^{*} \in \mathbf{R} \cup \infty .
$$

Thanks to functions of replication of genes, molecular lives are very active during its total life. Here we will introduce infinitely many reflectivity on mdna sequences which represents possibility of replication. There are sufficiently many common operations which are shared among all lives. The aim here is, first to formulate these operations whose results are also required to be infinitely many reflective. Then after one will try to reconstruct some metabolisms, in particular Glycolysis, using particular choice of $X$, consisted by shift operators and projections. A basic problem will be to seek for primitive elements (metabolism) which can grow to Glycolysis. More general construction and analysis of the energy will be in the next paper.

The content is as follows:

(1) M-dna sequences

(2) Mutation

(3) Replication

(4) Ensymes

(5) Selective splicing

(6) Converence and divergence

(7) Cancering

(8) Metabolism

(9) Conservative systems in metablisms

(10) Glycolysis

(11) Loop structure

The author has been very much influenced by a succesive seminar:

Initiation à la genomique fonctionnelle

: Aspects biologiques, mathematiques et algorithmiques

during 2000-2001 at the institut Henri Poincar'e. He would like to thank to the organizers of the seminar. In particular he would like to express his gratitude to Professor Misha Gromov for encouragement, from whom he has received many stimulation. He also would be thankful to IHES for their hospitarity. 


\section{M-DNA SEQUENCES}

Let $A$ be a (non commutative) algebra with involution *. Let us choose subsets:

(1) $I=\left\{s_{1}, \ldots, s_{m}\right\} \subset A$ and

(2) $X=\left\{X_{1}, \ldots, X_{n}, \ldots\right\} \subset A$. We say that $I$ is the initial sets and $X$ is the primary sets. We will say that $(A, I, X)$ as a $m$-dna data.

A $m$-dna sequence is a finite length string $V=X_{1} X_{2} \ldots X_{l}$, where $X_{i}$ are in $X$ with extra structures described below. Both sides of each $X_{i}$ are assigned with elements $a_{i}$ and $a_{i+1}$ in $A$ and $a_{1} \in I$ which satisfy the shift relation:

$$
X_{1}=a_{1} a_{2}^{*}, \quad \ldots \quad X_{i}=a_{i} a_{i+1}^{*}, \quad \ldots
$$

We will denote these data as:

$$
V=a_{1}\left[X_{1}\right] a_{2}\left[X_{2}\right] \ldots a_{l}\left[X_{l}\right] a_{l+1} .
$$

Usually one assumes $I=\{i d\}$.

Let $W=X_{1} \ldots X_{l}$ be a string which are assigned with only a sequence of primary sets. Then one can assign $\mathrm{m}$-dna sequences $V=a_{1}\left[X_{1}\right] a_{2}\left[X_{2}\right] \ldots a_{l}\left[X_{l}\right] a_{l+1}$ if there exists a family $\left\{a_{i}\right\}_{i}$ satisfying the shift relation. We will denote the set of m-dna sequences with respect to $W$ as:

$$
V(W, A)=\left\{V=a_{1}\left[X_{1}\right] a_{2}\left[X_{2}\right] \ldots a_{l}\left[X_{l}\right] a_{l+1}: \text { m-dna sequence }\right\} .
$$

We will say $W=X_{1} \ldots X_{l}$ is a primary sequence with respect to $V$.

Example 1.A: Let $V=a_{1}\left|X_{1}\right| a_{2}|\ldots| X_{l} \mid a_{l+1}$ be a m-dna sequence. Then $V^{*} \equiv a_{l+1}\left|X_{l}^{*}\right| a_{l}\left|\ldots a_{2}\right| X_{1}^{*} \mid a_{1}$ is also a m-dna sequence. We call this assignment $V \rightarrow V^{*}$ as an involution. We will say that $V$ is palindrome, if $V=V^{*}$.

\section{Mutation}

2.A Point mutation: Let $V=a_{1}\left|X_{1}\right| a_{2} \ldots a_{l}\left|X_{l}\right| a_{l+1}$ be a m-dna sequence. A point mutation of $V$ is another m-dna sequence:

$$
V^{\prime}=a_{1}^{\prime}\left|X_{1} \ldots a_{k-1}^{\prime}\right| a_{k}^{\prime}\left|X_{k}^{\prime}\right| a_{k+1}^{\prime} \mid X_{k+1} \ldots a_{l+1}^{\prime}
$$

( $V^{\prime}$ differes from $V$ in primary sequences only by replacing $X_{k}$ by another $X_{k}^{\prime}$ ). 
Example 2.A: Let $P$ and $P^{\prime}$ be projections on a Hilbert space where the equality $P P^{\prime}=P^{\prime}$ holds. Then $V=i d[i d] i d[P] P$ is a m-dna sequence. $V^{\prime}=$ $i d[i d] i d\left[P^{\prime}\right] P^{\prime}$ is a mutation of $V$.

2.B Intron: Let $V$ be a m-dna sequence. Supose the string splits into three parts:

$$
\begin{aligned}
V= & V_{1} \cup V_{2} \cup V_{3} \\
= & a_{1}\left|X_{1}\right| a_{2}\left|X_{2} \ldots a_{l_{1}}\right| X_{l_{1}}\left|a_{l_{1}+1} \cup a_{l_{1}+1}\right| X_{l_{1}+1}\left|a_{l_{1}+2}\right| X_{l_{1}+2} \ldots a_{l_{2}}\left|X_{l_{2}}\right| a_{l_{2}+1} \\
& \cup a_{l_{2}+1}\left|X_{l_{2}+1}\right| a_{l_{2}+2}\left|X_{l_{2}+2} \ldots a_{l}\right| X_{l} \mid a_{l+1} .
\end{aligned}
$$

An elimination of $V_{2}$ from $V$ consists of another m-dna sequence:

$$
V_{1} \cup V_{3}=a_{1}^{\prime}\left|X_{1}\right| a_{2}^{\prime}\left|X_{2} \ldots a_{l_{1}}^{\prime}\right| X_{l_{1}} X_{l_{2}+1}\left|a_{l_{2}+2}^{\prime}\right| X_{l_{2}+2} \ldots a_{l}^{\prime}\left|X_{l}\right| a_{l+1}^{\prime} \text {. }
$$

Let us say that for a m-dna sequence $V, V_{2}$ is a pre-intron, if $V_{1} \cup V_{3}$ exists. $V_{1} \cup V_{3}$ is called as a spliced $m$-dna sequence.

2.C Amplification: Let us take m-dna sequences $V=a_{1}\left|X_{1}\right| a_{2} \ldots a_{l-1}\left|X_{l-1}\right| a_{l}$ and $V^{\prime}=b_{1}\left[Y_{1}\right] b_{2}\left[Y_{2}\right] \ldots\left[Y_{k}\right] b_{k+1}$. Suppose that there is a primary element $Y$ such that:

$$
V[Y] V^{\prime}=a_{1}^{\prime}\left[X_{1}\right] a_{2}^{\prime} \ldots a_{l-1}^{\prime}\left[X_{l-1}\right] a_{l}^{\prime}[Y] b_{1}^{\prime}\left[Y_{1}\right] b_{2} \ldots b_{k-1}^{\prime}\left[Y_{k-1}\right] b_{k}^{\prime}
$$

consists of also a m-dna sequence. We will say that $V[Y] V^{\prime}$ is an amplification and $Y$ is a connecting element.

Let us take two m-dna sequences $V$ and $V^{\prime}$. Suppose there is connecting element $Y$ which makes an amplification $V|Y| V^{\prime}$. We will say that this mutation is non commutative, if $V^{\prime}|Y| V$ does not admit any m-dna sequence.

Example 2.C: Let $P, P^{\prime}, P^{\prime \prime}$ be projections satisfying $P P^{\prime}=P^{\prime}$ and $P^{\prime} P^{\prime \prime}=$ $P^{\prime \prime}$. Let us consider:

$$
\begin{aligned}
& V=i d|P| P, \quad V^{\prime}=i d\left|P^{\prime \prime}\right| P^{\prime \prime}, \quad Y=i d\left|P^{\prime}\right| P^{\prime}, \\
& V|Y| V^{\prime}=i d[P] P\left[P^{\prime}\right] P^{\prime}\left[P^{\prime \prime}\right] P^{\prime \prime}, \quad V^{\prime}|Y| V=i d\left[P^{\prime \prime}\right] P^{\prime \prime}\left[P^{\prime}\right] P^{\prime}[P] P .
\end{aligned}
$$

$V|Y| V^{\prime}$ satisfies shift relation, but $V^{\prime}\left|Y^{\prime}\right| V$ does not when $P^{\prime \prime} \neq P^{\prime}$.

2.D Frame shift: Let $V=a_{1}\left|X_{1}\right| a_{2}\left|\ldots a_{l}\right| X_{l} \mid a_{l+1}$ be a m-dna sequence, and $u \in A$ be a unitary, $u u^{*}=$ id. Then $V(u)=a_{2} u\left|X_{1}\right| a_{3} u|\ldots| a_{l} u\left|X_{l-1}\right| a_{l+1} u$ is 
also a m-dna sequence, and we will say that $V^{\prime}$ is a frame shift of $V$.

Example 2.D: Let $V=a_{1}\left|X_{1}\right| a_{2}\left|\ldots a_{l}\right| X_{l} \mid a_{l+1}$ be a m-dna sequence, and take a unitary $\sigma \in A$ with $\sigma^{3}=\mathrm{id}$. Then one considers another m-dna sequence:

$$
\begin{gathered}
\tilde{V}=\left(\begin{array}{cc}
\sigma & 0 \\
0 & a_{1}
\end{array}\right)\left[\left(\begin{array}{cc}
\sigma^{*} & 0 \\
0 & X_{1}
\end{array}\right)\right]\left(\begin{array}{cc}
\sigma^{2} & 0 \\
0 & a_{2}
\end{array}\right)\left[\left(\begin{array}{cc}
\sigma^{*} & 0 \\
0 & X_{2}
\end{array}\right)\right]\left(\begin{array}{cc}
1 & 0 \\
0 & a_{3}
\end{array}\right) \\
\left.\left[\left(\begin{array}{cc}
\sigma^{*} & 0 \\
0 & X_{3}
\end{array}\right)\right]\left(\begin{array}{cc}
\sigma & 0 \\
0 & a_{4}
\end{array}\right)\left[\left(\begin{array}{cc}
\sigma^{*} & 0 \\
0 & X_{4}
\end{array}\right)\right]\left(\begin{array}{cc}
\sigma^{2} & 0 \\
0 & a_{5}
\end{array}\right)\left[\begin{array}{cc}
\sigma^{*} & 0 \\
0 & X_{5}
\end{array}\right)\right] \ldots
\end{gathered}
$$

Now let us choose $u=\left(\begin{array}{ll}\sigma & 0 \\ 0 & 1\end{array}\right)$. Then $\tilde{V}(u)$ expresses frame shift on codon correspondence between DNA (RNA) and amino-acids.

2.E Inequal crossing over: Let $V=V_{1} V_{2}$ and $V^{\prime}=V_{1}^{\prime} V_{2}^{\prime}$ be two m-dna sequences with primary sequences as $\left(X_{1}\left({ }^{\prime}\right), \ldots, X_{l\left(^{\prime}\right)}\left({ }^{\prime}\right), Y_{1}\left({ }^{\prime}\right), \ldots, Y_{k\left({ }^{\prime}\right)}\left({ }^{\prime}\right)\right)$ respectively. If both of another primary sequences $V_{1} V_{2}^{\prime}=\left(X_{1}, \ldots, X_{l}, Y_{1}^{\prime}, \ldots, Y_{k^{\prime}}^{\prime}\right)$ and $V_{1}^{\prime} V_{2}=\left(X_{1}^{\prime}, \ldots, X_{l^{\prime}}^{\prime}, Y_{1}, \ldots, Y_{k}\right)$ also admit m-dna sequences $U$ and $U^{\prime}$ respectively, then we will say that $U$ and $U^{\prime}$ as inequal crossing over of $V$ and $V^{\prime}$.

2.F System of mutations: A system of mutation is a finite set $\mathfrak{S}$ where any element $s \in \mathfrak{S}$ represents some operation described from 2.A to 2.E. Let $\bar{V}$ be a set of m-dna sequences. Then $\mathfrak{S}(\bar{V})$ gives another set of $\mathrm{m}$-dna sequences. One may iterate this process as:

$$
\bar{V} \subset \mathfrak{S}(\bar{V}) \subset \mathfrak{S}^{2}(\bar{V}) \subset \mathfrak{S}^{3}(\bar{V}) \subset \ldots
$$

This will represent a simple model of evolution.

Example 2.F: Let $M_{n}(\mathbf{C})$ be the set of $n \times n$ complex matrices, and put $M_{\infty}(\mathbf{C})=\lim _{n} M_{n}(\mathbf{C})$. Suppose the primary sets $X=\left\{X_{1}, \ldots, X_{n}, \ldots\right\} \subset$ $M_{\infty}(\mathbf{C})$. Then we will call $X$ as a finite type. Any set $\bar{V}$ whose primary sets consistes of finite type will be also called as finite type.

Let $\mathfrak{S}$ be the set of 2.A,2.B, 2.C,2.E. Then $N$-th stage of evolution of $\bar{V}$ is also of finite type for any $N \geq 0$. 


\section{ReplicAtion}

3.A Replication: Suppose the algebra $A$ is a closed $*$ subalgebra of $B(H)$, where $H$ is a separable Hilbert space. Let us say that an operator $a \in A$ is replicable, if there is a unitary operator $U: H \cong H \oplus H$ satisfying:

$$
U a U^{*}=\left(\begin{array}{cc}
a^{\prime} & 0 \\
0 & a^{\prime \prime}
\end{array}\right) .
$$

We will say that $a^{\prime \prime}$ is a reflective element. Reflective elements are not unique. Let $X=\left\{X_{1}, \ldots, X_{l}, \ldots\right\}$ be the primary set. In the following, we will assume that $X$ is closed under replication, in the sense that any $X_{i}$ has a unitary $U$ satisfying $U X_{i} U^{*}=\operatorname{diag}\left(X_{j}, X_{k}\right)$ for some $j, k$.

Let us take a m-dna data $(A, I, X)$ where $A$ is as above, and $I$ and $X$ are both consisted by replicable elements. Let us denote by $Y_{i}$ as reflective elements of $X_{i}$. Let us take a m-dna sequence $V=a_{1}\left|X_{1}\right| a_{2}\left|X_{2} \ldots a_{l}\right| X_{l} \mid a_{l+1}$. A replication of $V$ is another m-dna sequence $b_{1}\left|Y_{1}\right| b_{2}\left|\ldots b_{l}\right| Y_{l} \mid b_{l+1}$ where each $Y_{i}$ is a reflective element of $X_{i}$, using the same unitary $U$ for all $i$.

Remark 3.A: (1) Suppose there are no invariant subspace for $a \in A$, and so no non trivial splitting as above. In this case, one replaces $a$ by $\operatorname{diag}(a, a, \ldots)$ acting on $H \oplus H \oplus \ldots$ Then the latter splits as $\operatorname{diag}(a, a, \ldots) \oplus \operatorname{diag}(a, a, \ldots)$. Then even though $\operatorname{diag}(a, a, \ldots)$ contains the same information as $a$, this allows to make replication. (but the algebra $A$ becomes much larger). In later examples, one assumes when a primary sequence $\left(X_{1}, \ldots, X_{l}\right)$ admits a m-dna sequence, then it always can make replication.

(2) Here one will only consider single sequences, even though in practice when genes make replication, the double helix structure plays an important role.

Example 3.A: (1) Let us take an orthnormal basis $H=\left\{v_{0}, v_{1}, v_{2}, \ldots\right\}$ and an unitary $U_{0}\left(v_{2 i}\right)=v_{i}+0, U_{0}\left(v_{2 i+1}\right)=0+v_{i} \in H \oplus H$. Then $R: v_{i} \rightarrow v_{i+2}$ is replicable. 
(2) Let us modify $U_{0}$ above slightly, and define $U_{0}(2 i, 0)$ by the following:

$$
U_{0}(2 i, 0)\left(v_{k}\right)= \begin{cases}v_{2 i}+0, & k=2 i, \\ v_{i}+0, & k=4 i, \\ v_{j}+0, & k=2 j, j \neq i, 2 i, \\ 0+v_{j}, & k=2 j+1 .\end{cases}
$$

Then $U_{0}(2 i, 0)$ are all unitary and satisfy $U_{0}(2 i, 0)\left(v_{2 i}\right)=v_{2 i}$. Let $P(2 i, 0)$ be the projection to $v_{2 i}, P(2 i, 0)\left(v_{2 i}\right)=v_{2 i}$. Then clearly $P(2 i, 0)$ are all replicable. One can obtain $P(i, j)$ similarly.

(3) Let us take a Hilbert space $H$ and its basis $\left\{\ldots, v_{-l}, \ldots, v_{0}, v_{1}, v_{2}, \ldots\right\}$. Let us put shift operators as follows:

$$
Q:\left\{\begin{array}{l}
v_{2 i+1} \mapsto v_{2 i+3} \\
v_{2 i} \mapsto v_{2 i+4}
\end{array}, \quad P: v_{i} \mapsto v_{i+1}, \quad R=P^{2}: v_{i} \mapsto v_{i+2}\right.
$$

Let us consider two m-dna sequences:

$$
\text { (1) } i d\left[P^{*}\right] P[Q] Q^{\prime}, \quad(2) \quad i d\left[R^{*}\right] R[Q] R^{\prime} .
$$

Using $U_{0}$ above, these have replications as follows:

$$
\text { (1) }\left(\begin{array}{ll}
1 & 0 \\
0 & 1
\end{array}\right)\left[\begin{array}{cc}
0 & 1 \\
P^{*} & 0
\end{array}\right]\left(\begin{array}{cc}
0 & P \\
1 & 0
\end{array}\right)\left[\begin{array}{cc}
R & 0 \\
0 & P
\end{array}\right]\left(\begin{array}{cc}
0 & P^{*} \\
P^{*} & 0
\end{array}\right) \text {. }
$$

Thus (1) is not reflective. For (2), we have a splitting:

$$
\left(\begin{array}{ll}
1 & 0 \\
0 & 1
\end{array}\right)\left[\begin{array}{cc}
P^{*} & 0 \\
0 & P^{*}
\end{array}\right]\left(\begin{array}{cc}
P & 0 \\
0 & P
\end{array}\right)\left[\begin{array}{cc}
R & 0 \\
0 & P
\end{array}\right]\left(\begin{array}{cc}
P^{*} & 0 \\
0 & 1
\end{array}\right)
$$

Thus the reflective element is $i d\left[P^{*}\right] P[P] i d$ (parindrome) which also consists of a m-dna sequence. The second stage of replication is as follows:

$$
(2)^{\prime} \quad\left(\begin{array}{ll}
1 & 0 \\
0 & 1
\end{array}\right)\left[\begin{array}{cc}
0 & 1 \\
P^{*} & 0
\end{array}\right]\left(\begin{array}{ll}
0 & P \\
1 & 0
\end{array}\right)\left[\begin{array}{ll}
0 & P \\
1 & 0
\end{array}\right]\left(\begin{array}{ll}
1 & 0 \\
0 & 1
\end{array}\right)
$$

Thus the above reflective element is not secondary reflective. Thus the sequence (1) cannot replicate. (2) can do only once.

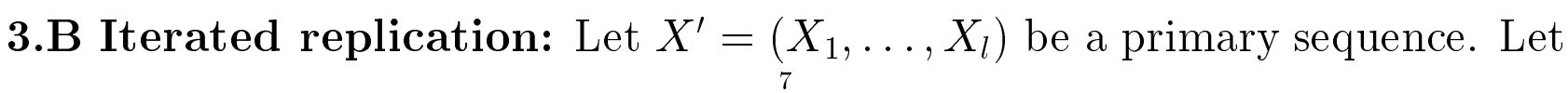


us choose an isomorphism $U: H \cong H \oplus H$ with $U X_{i} U^{*}=\operatorname{diag}\left(X_{i}(1)^{\prime}, X_{i}(1)\right)$. If one can find another unitary $U_{1}$ as above with a decomposition $U_{1} X_{i}(1)\left(U_{1}\right)^{*}=$ $\operatorname{diag}\left(X_{i}(2)^{\prime}, X_{i}(2)\right)$, we say that $X_{i}(2)$ is a secondary reflection element. One may iterate this process, and find seccesively $U_{2}, U_{3}, \ldots$ and $X_{i}(2), X_{i}(3), \ldots$

Recall $V\left(X^{\prime}, A\right)$ in section 1 . Let $V=a_{1}\left|X_{1}\right| a_{2} \ldots a_{l}\left|X_{l}\right| a_{l+1}$ be a m-dna sequence. Then $V$ is infinitely many reflective, if $V\left(\left(X_{1}(i), \ldots, X_{l}(i)\right), A\right)$ are non empty for all $i$.

Example 3.B: Let us choose arbitrarilry a family $\left\{X_{0}, X_{1}, \ldots\right\} \subset X$, choose any isomorphism $H \cong$ closure $\oplus_{i=0}^{\infty} H_{i}$ where $H_{i}$ are all the same Hilbert spaces. Passing through this, one obtains an operator $X^{\prime}=\operatorname{diag}\left(X_{0}, X_{1}, \ldots\right)$ acting on $H$. Then one naturally gets a reflective element $X_{1}^{\prime} \equiv \operatorname{diag}\left(X_{1}, X_{2}, \ldots\right)$. As a secondary reflective element, one gets $X_{2}^{\prime} \equiv \operatorname{diag}\left(X_{2}, X_{3}, \ldots\right)$. Iterating one obtains $n$-th reflective element $X_{n}^{\prime} \equiv \operatorname{diag}\left(X_{n}, X_{n+1}, \ldots\right)$. Thus the realizing problem of reflective elements has a trivial solution.

Similarly choose any family $\left\{\ldots, X_{-l}, \ldots, X_{0}, X_{1}, \ldots\right\}$ and an isomorphism $H \cong$ closure $\oplus_{i=-\infty}^{\infty} H_{i}$. In the both sides case also, the realizing problem has a trivial solution, from an operator $X^{\prime \prime}=\operatorname{diag}\left(\ldots, X_{0}, X_{1}, \ldots\right)$.

Let us take a unitary $U: H \cong H=$ closure $\oplus H_{i}$, and choose any vectors $v_{i} \in H_{i}$ and $v_{j} \in H_{j}, i<j$. Then we will say that $U$ preserves the order on $\left(v_{i}, v_{j}\right)$, if there is a pair $i^{\prime}<j^{\prime}$ with $U\left(v_{i}\right) \in H_{i^{\prime}}$ and $U\left(v_{j}\right) \in H_{j^{\prime}}$.

Now let $\left\{\ldots, X_{-l}, \ldots, X_{0}, X_{1}, \ldots\right\}$ be as above, and consider one side and both sides sequences:

$$
\begin{aligned}
& X^{\prime}=\left(X_{i_{0}}, X_{i_{1}}, \ldots\right) \in B(H)=B\left(\text { closure } \oplus_{i=0}^{\infty} H_{i}\right), \\
& X^{\prime \prime}=\left(\ldots, X_{i_{-l}}, \ldots, X_{i_{0}}, X_{i_{1}}, \ldots\right) \in B(H)=B\left(\text { closure } \oplus_{i=-\infty}^{\infty} H_{i}\right) .
\end{aligned}
$$

where the indices $I^{\prime}=\left\{i_{0}, i_{1} \ldots\right\}, I^{\prime \prime}=\left\{\ldots, i_{-l}, \ldots, i_{0}, i_{1}, \ldots\right\} \subset\{0,1,2, \ldots\}$ satisfy the followings:

(1) each $l \in\{0,1,2, \ldots\}$ appears infinitely many times in both of $I^{\prime}$ and $I^{\prime \prime}$ in the positive direction, and

(2) for a sufficiently large $j_{0}, i=0$ for all $i \leq-j_{0}$ in $I^{\prime \prime}$.

Let us consider a problem to seek for a unitary $U: H \cong H$ satisfying the following: 
(1) $U^{*} X^{\prime} U=\operatorname{diag}\left(X_{0}^{\prime}, X_{1}^{\prime}\right)$ where $X_{0}$ does not appear in $X_{1}^{\prime}$,

(2) $X_{0}^{\prime}=X^{\prime}$, and

(3)for any $i_{l}, i_{k}$ in $X_{m}^{\prime}$ component, $U$ preserves the order between $H_{i_{l}}$ and $H_{i_{k}}$ for $m=0,1$ respectively.

We will call such an operator as an order preserving separating unitary. Notice that once a family $\left\{X_{i}\right\}_{i}$ is fixed, then $X^{\prime}$ and $X^{\prime \prime}$ are expressed as one side or both sides sequences by $\{0,1, \ldots\}$ respectively.

An unexpected answer is that (1) in one side case, there are always no order preserving separating unitary, and (2) in both sides case, it always exists. In terms of an expression of numbers, this is the following lemma:

Lemma 3.B: Let $X^{\prime}=\left(x_{0}, x_{1}, \ldots\right)$ be a one side sequence in $\{0,1, \ldots\}$. Then there are no division of $X^{\prime}$ into two sequences $X^{\prime}=Y \cup Z\left(Y=\left(x_{m(l)}\right)\right.$ and $Z=\left(x_{n(l)}\right)$ with $\left.\mathbf{N}=\{m(l)\}_{l} \cup\{n(l)\}_{l}\right)$ such that

(1) both of $Y$ and $Z$ consist of infinite sequences and they are order preserving,

(2) $Y=X^{\prime}$ as sequences and (3) $Z$ does not contain 0 .

Let $X^{\prime \prime}$ be a both side sequence. Then there is a division of $X^{\prime \prime}$ into two sequences satisfying (1), (2) and (3) above.

Proof: This can be seen from the next two examples. Let us consider $X^{\prime \prime}=$ $(\ldots, 0,0,0,1,0,1,2,0,1,2,3, \ldots)$. Then one can divide as:

$$
X^{\prime \prime}=(\ldots, 0,0,1,0,1,2,0,1,2,3, \ldots) \cup(1,2,3, \ldots) .
$$

In the case $X^{\prime}=(0,0,1,0,1,2,0,1,2,3, \ldots)$, there are no such division.

Notice that automorphism groups of one or two sides subshift of finite types have mutually very different structures $([\mathrm{K}])$.

\section{Ensyme}

Ensymes play roles to activate or disactivate functions of genes. They can find particular genes to which they catalyze. Then a particular domain of ensymes (activating domain) attaches to domains of genes. For this, higher dimensional structures of genes are very important. 
Let us take two m-dna sequences $V=a_{1}\left|X_{1}\right| a_{2}\left|\ldots a_{l}\right| X_{l} \mid a_{l+1}$ and $W=$ $b_{1}\left|Y_{1}\right| b_{2}\left|\ldots b_{k}\right| Y_{k} \mid b_{k+1}$. Let us say that $W$ catalyzes (as an ensyme) on $V$ (m-dna sequence) at $(i, j)$, if the primary sequence:

$$
V[W] \equiv\left(X_{1}, \ldots \ldots X_{i-1}, X_{i} Y_{j}, X_{i+1}, \ldots X_{l}\right)
$$

posesses another m-dna sequence. Namely there is $\left(a_{1}^{\prime}, \ldots, a_{l+1}^{\prime}\right)$ with:

$$
a_{1}^{\prime}\left|X_{1}\right| \ldots a_{l-1}^{\prime}\left|X_{l-1}\right| a_{l}^{\prime}\left|X_{i} Y_{j}\right| a_{i+1}^{\prime}\left|X_{i+1}\right| \ldots\left|a_{l}^{\prime}\right| X_{l} \mid a_{l+1}^{\prime}
$$

consists of a m-dna sequence.

4.A Inversible ensymes: Let $V$ and $V^{\prime}$ be two m-dna sequences. If there are ensymes $W$ and $W^{\prime}$ with the equalities:

$$
V[W]=V^{\prime}, \quad V^{\prime}\left[W^{\prime}\right]=V
$$

then we will say that $W$ and $W^{\prime}$ are mutually inverse ensymes w.r.t. $\left(V, V^{\prime}\right)$.

Example 4.A: Let $H=\left\{v_{0}, v_{1}, \ldots\right\}$ be a Hilbert space. Let us take $S$, the standard shift $S: v_{i} \mapsto v_{i+1}$ and $P$, the projection as $P\left(v_{i}\right)=v_{i}, i \neq 0,1$ and $P\left(v_{0}\right)=P\left(v_{1}\right)=0$. Let us take two m-dna sequences:

$$
V=i d[P S](P S)^{*}, \quad V^{\prime}=i d\left[S^{2}\right]\left(S^{2}\right)^{*} .
$$

Notice the equality $P S^{2}=S^{2}$. Then one can find:

$$
W=i d[S] S, \quad W^{\prime}:\left\{\begin{array}{l}
v_{0} \rightarrow 0 \\
v_{i} \rightarrow v_{i-1}, \quad i \geq 1 .
\end{array}\right.
$$

These give mutually inversible ensymes.

Notice that the primary structure of $V[W]$ also differs from that of $V$. After $W$ has stopped catalyzing, $V[W]$ will return to the original $V$. So in practice catalyzing should be inversible.

4.B Promotor: Let $V_{0}=a_{0}\left|X_{0}\right| a_{1}\left|X_{1}\right| a_{2}|\ldots| a_{l-1}\left|X_{l-1}\right| a_{l}$ be a sequence whose primary sequence does not admit any m-dna sequences. Let $W=i d|Y| Y^{*}$ be a m-dna sequence so that the primary sequence $\left(X_{0} Y, X_{1}, \ldots, X_{l-1}\right)$ has m-dna sequences. Thus $V_{0}$ cannot replicate by itself. After catalyzed by $W, V=V_{0}[W]$ becomes a m-dna sequence and begins replicating. One will call $V_{0}$ as a pre $m$ dna sequence. $X_{0}$ is called a promotor. 
Example 4.B: Let $H=\left\{\ldots, v_{-l}, \ldots, v_{0}, \ldots, v_{l}, \ldots\right\}$ be a Hilbert space, $Q(i)$ and $Q(i, j)$ be the projections on $\left\{v_{i}\right\}$ and $\left\{v_{i}, v_{j}\right\}$ respectively. $P(i) \equiv i d-Q(i)$.

Let us consider a pre m-dna sequence $V_{0}=i d[i d] i d[Q(1)] Q(1)[Q(1,2)] Q(1,2)$ and take $W=i d[P(2)] P(2)$. Then the primary sequence $(P(2), Q(1), Q(1,2))$ has a m-dna sequence $i d[P(2)] P(2)[Q(1)] Q(1,2)[Q(1,2)] i d$.

4.C Mutually exclusive promotors: Let $V=a_{1}\left|X_{1}\right| a_{2}\left|\ldots a_{l}\right| X_{l} \mid a_{l+1}$ be a pre m-dna sequence. Suppose $V$ posseses two promotors at $X_{i}$ and $X_{j}$ such that two ensymes $Y_{i}$ and $Y_{j}$ catalyze on these regions respectively.

If the following conditions are satisfied, then these two promotors are said to be mutually exclusive;

(1) both of $V\left[Y_{i}\right]$ and $V\left[Y_{j}\right]$ are infinitely many reflective,

(2) $V\left[Y_{i}\right]\left[Y_{j}\right]$ is only finitely many reflective.

Examples 4.C: Let $P\left(i_{1}, \ldots, i_{N}\right)$ be the corank $N$ projection by:

$$
P\left(i_{1}, \ldots, i_{N}\right)\left(v_{l}\right)= \begin{cases}0 & l=i_{j}, \\ v_{l} & \text { others }\end{cases}
$$

One puts $Q\left(i_{1}, \ldots, i_{N}\right)=1-P\left(i_{1}, \ldots, i_{N}\right)$. Moreover let $S$ be the shift by $v_{i} \mapsto v_{i+1}$. Then one considers the primary sequence:

$$
X=(P(1), P(0,1), P(-1,1)) .
$$

$V(X, A)$ is empty. Let us choose $Y_{1}=Y_{2}=S$. Then one has three primary sequences:

$$
\begin{aligned}
& X_{1}=(P(1) S, P(0,1), P(-1,1)), \quad X_{2}=(P(1), P(0,1) S, P(-1,1)), \\
& X_{3}=(P(1) S, P(0,1) S, P(-1,1)) .
\end{aligned}
$$

$V\left(X_{1}, A\right)$ and $V\left(X_{2}, A\right)$ are both non empty, on the other hand $V\left(X_{3}, A\right)$ is empty as shown below:

$$
\begin{aligned}
& i d[P(1) S] S^{-1} P(1)[P(0,1)] S^{-1} P(2)[P(-1,1)] S^{-1} P(0) \in V\left(X_{1}, A\right), \\
& i d[P(1)] P(1)[P(0,1) S] S^{-1} P(0)[P(-1,1)] S^{-1} P(2) \in V\left(X_{2}, A\right), \\
& i d[P(1) S] S^{-1} P(1)[P(0,1) S] S_{11}^{-2} P(2)[P(-1,1)] * .
\end{aligned}
$$


One can see there are no $a$ with $S^{-2} P(2) a=P(-1,1)$ which is equivalent to $P(2) a=S^{2} P(-1,1)$, since the r.h.s. contains non zero $v_{2}$ component in the image, on the other hand the l.h.s. does not for any $a$.

In practice, many mutually exclusive promotors are found, e.g., the switching of grobin genes.

4.D Succesive ensymes: Let $V=a_{1}\left|X_{1}\right| a_{2}\left|\ldots a_{l}\right| X_{l} \mid a_{l+1}$ be a m-dna sequence, and consider the primary sequence $\left(X_{1}, \ldots, X_{l}\right)$. Suppose there are two states $Y_{i}$ and $Y_{j}$ catalyzing on $V$ respectively.

If the following conditions are satisfied, then these two catalyzing processes are said to be succesive;

(1) $V\left[Y_{1}\right]$ and $V\left[Y_{j}\right]$ are only finitely many reflective, and

(2) $V\left[Y_{i}\right]\left[Y_{j}\right]$ is infinitely many reflective.

Example 4.D: Let $P\left(i_{1}, \ldots, i_{N}\right)$ and $Q\left(i_{1}, \ldots, i_{N}\right)$ be as before. First one considers a simple case. Let us consider a m-dna sequence:

$$
V=i d[i d] i d[P(2)] P(2)[Q(1)] Q(1) .
$$

Then one chooses two ensymes:

$$
Y_{1}=P(1), \quad Y_{2}=P(1) .
$$

When these catalyze on $V$, then the corresponding primary sequences become as:

$$
\begin{gathered}
V\left[Y_{1}\right]=(P(1), P(2), Q(1)), \quad V\left[Y_{2}\right]=(i d, P(1,2), Q(1)), \\
V\left[Y_{1}\right]\left[Y_{2}\right]=(P(1), P(1,2), Q(1)) .
\end{gathered}
$$

Let $\left(a_{1}, a_{2}, a_{3}, a_{4}\right)$ be a sequence with $a_{1}=i d$. Let us try to find m-dna sequences $V=a_{1}|P(1)| a_{2}|P(2)| a_{3}|Q(1)| a_{4}, V^{\prime}=a_{1}|i d| a_{2}|P(1,2)| a_{3}|Q(1)| a_{4}$ and $V^{\prime \prime}=a_{1}|P(1)| a_{2}|P(1,2)| a_{3}|Q(1)| a_{4}$. $V^{\prime \prime}$ has a solution by:

$$
V^{\prime \prime}=i d|P(1)| P(1)|P(1,2)| P(2)|Q(1)| Q(1)
$$

One has partial solutions for:

$$
V=i d|P(1)| P(1)|P(2)| *, \quad V^{\prime}=i d|i d| i d|P(1,2)| P(1,2)|Q(1)| * .
$$

Here it is impossible to find $*$ to satisfy the shift relation. 
Extension 4.D: Let us take a Hilbert space $H^{\prime}=H_{1} \oplus H_{2} \oplus H_{3}$ where each $H_{i}$ is also an infinite dimensional Hilbert space. Let $P(i), i=1,2,3$, be the projections to $H_{i}$. Let us take another projection $P$ and a primary sequence $X=(P, 0, P(1))$, where the first two states are promotors as above. Then there is a m-dna sequence with this primary sequence, if and only if $P P(1)=P(1)$.

Notice that any projection with infinite dimensional kernel and range are all unitary equivalent. Thus there is a unitary $U$ satisfying:

$$
U^{*} P(1) U=\left(\begin{array}{cc}
P(1) & 0 \\
0 & P(1)
\end{array}\right)
$$

Let us take a family of unitaries $U_{i}, i=1,2, \ldots$ so that one gets inductively equations $U_{i}^{*} P_{i} U_{i}=\operatorname{diag}\left(P_{i}, P_{i+1}\right), P_{1}=P$. Let $\left(a_{1}^{i}, a_{2}^{i}, a_{3}^{i}, a_{4}^{i}\right)$ be a sequence with $a_{1}^{i}=i d$, and try to judge whether $V_{1}$ is infinitely many reflective, i.e., to find a family of m-dna sequences:

$$
V_{i}=a_{1}^{i}\left|P_{i}\right| a_{2}^{i}|0| a_{3}^{i}|P(1)| a_{4}^{i} .
$$

Then the following can be seen; $V_{1}$ is infinitely many reflective, if and only if $P(1) \subset \subset P_{i}$ are satisfied for all $i=1,2, \ldots$

4.E Switching, special cases: Let $P V$ be a pre m-dna sequence where two different ensymes $W$ and $W^{\prime}$ at $i$ and $(i, j)$, acts on the promotor $P$ which are mutually exclusive. Let us denote $P V(1)=P V[W]$ and $P V(2)=P V\left[W^{\prime}\right]$. Here one considers a special case of functions of $(P V, W)$ as follows:

(1) $W$ catalyzes on $P$ and produces $P V(1)$.

(2) $W^{\prime}=P V(1)$ catalyzes on $P$, and produces $P V\left[W^{\prime}\right]=P V(2)$.

Thus when $P V(1)$ has been produced too much, then $P V(2)$ also increases.

Example 4.E: Let $P\left(i_{1}, \ldots, i_{N}\right)$ and $Q\left(i_{1}, \ldots, i_{N}\right)$ be in 4.C. Then one considers a pre m-dna sequence $P V=i d[i d] i d[Q(1)] Q(1)[Q(1,2)] Q(1,2)$ and an ensyme $W=i d[P(2)] P(2)$. Then:

$$
W^{\prime}=i d[P(2)] P(2)[Q(1)] Q(1,2)[Q(1,2)] i d
$$


catalyzes on $P$ and produces

$$
\begin{aligned}
P V\left[W^{\prime}\right] & =i d[i d Q(1)] Q(1)[Q(1) Q(1,2)] Q(1,2)[Q(1,2)] i d \\
& =i d[Q(1)] Q(1)[Q(1)] Q(1,2)[Q(1,2)] i d
\end{aligned}
$$

4.F Switching: Let us take a pre m-dna sequence $V$, and ensymes $W, W^{\prime}, U, U^{\prime}$ with a diagram:

$$
\begin{gathered}
V \\
\uparrow \\
W-[U] \rightarrow W^{\prime}-\left[U^{\prime}\right] \rightarrow W
\end{gathered}
$$

Thus $U, U^{\prime}$ are ensymes which act on $W$ and $W^{\prime}$ as mutual inverses. $W^{\prime}$ can catalyze on $V$ and $W$ cannot.

4.G Relation with inequally crossing over: Let us take two m-dna sequences $V=V_{0} V_{1}$ and $U=U_{0} U_{1}$. Suppose the inequally crossing over occurs:

$$
(V, U) \mapsto\left(V_{0} U_{1}, V_{1} U_{0}\right) .
$$

This will produce new ensymes.

4.H Inactivating: Let us take a m-dna sequence $V$, and ensymes $W$ and $U, U^{\prime}$. suppose both of $U$ and $U^{\prime}$ catalyze on $W$ satisfying:

(1) $W[U]$ can catalyze on $V$,

(2) $W\left[U^{\prime}\right]$ cannot do it.

The ensyme $U^{\prime}$ is said to be inactivating.

4.I Indirect ensymes: Let us take two m-dna sequences $V$ and $W$. Suppose there is a cycle of catalyzing system:

$$
W-[V] \rightarrow W[V] \equiv W^{\prime}, \quad V-\left[W^{\prime}\right] \rightarrow V\left[W^{\prime}\right] \equiv V^{\prime} .
$$

Then we will say that $W$ is an indirect ensyme for this catalyzing system $V \rightarrow$ $V^{\prime}$.

4.J Rigidity: Let us fix a primary sequence $X=\left\{X_{1}, \ldots, X_{l}\right\}$. Recall $V(X, A)$ in section 1 . We will say that $X$ is rigid if there is exactly only one $V \in L(X, A)$ with $a_{1} \in I$ and $V=a_{1}\left|X_{1}\right| a_{2} \ldots\left|a_{l}\right| X_{l} \mid a_{l+1}$. 
In many of the examples here, the primary sets are rigid with $I=\{i d\}$.

Let us take a m-dna sequence $V=a_{1}\left|X_{1}\right| a_{2} \mid X_{2} \ldots$ and an ensyme $W=$ $b_{1}|Y| b_{2}$. We will say that $V$ is projectively rigid, if the following is satisfied; suppose $W$ catalyzes on $V$ at $i$. If $V[W]$ consists of a m-dna sequence, then one has the equality $X_{i}=X_{i} Y$.

Example 4.J: Let us put $H=H_{1} \oplus H_{2}$ and put $P_{i}$ as the projections on $H_{i}$. Let us take a m-dna sequence $V=i d\left[P_{1}\right] P_{1}\left[P_{1}\right] i d[i d] i d$. Let us take another projection $X$. Let us put $W=i d[X] X$ and catalyze $W$ on $i d\left[P_{1}\right] P_{1}$ factor in $V$. Then one has $V[W]=i d\left[P_{1} X\right] P_{1} X\left[P_{1}\right] i d[i d] i d$. Thus by the definition, if $V[W]$ consists of a m-dna sequence, then the equality $P_{1} X=P_{1}$ holds.

4.K Amplification: Let $P V$ be a m-dna sequence. Let us consider a pre m-dna sequence $P V V$.

Suppose that $V$ and $V V$ catalyze on $P$ as ensyme. If the following two conditions are satisfied, then the amplification $P V V$ will be called self controlling:

(1) $P V[V]$ is infinitely many reflective,

(2) $P V V[V V]$ is only finitely many reflective.

If the converse holds:

(1) $P V[V]$ is only finitely many reflective,

(2) $P V V[V V]$ is infinitely many reflective,

then $P V V$ will be called self amplification.

Example 4.K: Let us take a Hilbert space $H=H_{1} \oplus H_{2}$ and put the projections on $H_{i}$ by $P_{i}$. Let us consider m-dna sequences:

$$
P=i d|i d| i d|i d| i d, \quad V=i d\left|P_{1}\right| P_{1} .
$$

Then the followings show that $P V V$ is self amplification:

$$
\begin{aligned}
& P V=i d[i d] i d[i d] i d\left[P_{1}\right] P_{1}, \quad P V[V]=i d\left[P_{1}\right] P_{1}[i d] i d\left[P_{1}\right] P_{1}, \\
& P V V=i d[i d] i d[i d] i d\left[P_{1}\right] P_{1}\left[P_{1}\right] P_{1}, \quad P V V[V V]=i d\left[P_{1}\right] P_{1}\left[P_{1}\right] P_{1}\left[P_{1}\right] P_{1}\left[P_{1}\right] P_{1} .
\end{aligned}
$$

\section{Selective SPlicing}

Let $V=P V_{1} V_{2}$ be a (pre) m-dna sequence. If there is an ensyme $W$ so that $V[W]$ has a maximal sequence $V_{1}$ of infinitely many reflection, then the 
procedure $V \rightarrow V[W] \rightarrow V_{1}$ is called splicing.

Example 5.A: Let us put $H=H_{1} \oplus H_{2} \oplus H_{3}$ where $H_{i}$ are infinite dimensional Hilbert spaces. Let us denote by $P$ and $P^{\prime}$ as the projections on $H_{1} \oplus H_{2}$ and on $H_{1}$ respectively. Let $V=P_{1} V_{1} P_{2} V_{2}$ be a m-dna sequence, where:

$$
P_{1}=i d|P| P, \quad V_{1}=P|P| i d, \quad P_{2}=i d|i d| i d, \quad V_{2}=i d|i d| i d .
$$

Let us take an ensyme $W=i d\left|P^{\prime}\right| P^{\prime}$ and catalyze it on $P_{2}$. Then as a maximal sequence, one gets the following:

$$
V^{\prime}=i d[P] P[P] i d\left[P^{\prime}\right] P^{\prime}
$$

5.B Splicing systems: Let $V=P V_{1} \ldots V_{l}$ be a (pre) m-dna sequence. Suppose there are subindices $\left\{m_{1}, \ldots, m_{k}\right\} \subset\{1, \ldots, l\}$ so that each $V_{m_{j}}$ is also infinitely many reflective. We will say that a family of ensymes $\left\{W_{1}, \ldots, W_{k}\right\}$ splices $\left\{V_{m_{j}}\right\}_{j}$, if each $W_{j}$ splices $V_{m_{j}}$.

Let $V=P V_{1} V_{1}^{\prime} V_{2} V_{2}^{\prime} \ldots V_{l} V_{l}^{\prime}$ be a m-dna sequence. A succesive splicing on $V$ is a system of splicing as follows; there is an ensyme $W$ catalyzing on $P$, and replicating $V_{1}$. Then $V_{1}$ again catalyzing on $P$, and replicating $V_{2}$. One can continue this process until replicating $V_{l}$.

5.C Self splicing systems: Let $V=a_{1}\left|X_{1}\right| a_{2} \ldots$ be a (pre) m-dna sequence. Suppose an ensyme $W$ catalyzes on $V, V_{1}=V[W]$ and it produces $W_{1}$ by splicing. Next let us catalyze $W_{1}$ on $V_{1}$ catalyzing as an ensyme, and produces $W_{2}$. Similarly let us catalyze $W_{2}$ on $V_{2}$ and produce $W_{3}$. Succesively one may obtain $\left(V_{l}, W_{l}\right), l=1,2, \ldots$ This process will be called as a self splicing system.

Example 5.C: Let us define shifts $S(n)$ and projections $P(n)$ as follows:

$$
S(n):\left\{\begin{array}{l}
v_{2^{n} k} \rightarrow v_{2^{n+1} k} \\
v_{i} \rightarrow v_{i}, \quad i \neq 2^{n} k
\end{array} \quad P(n):\left\{\begin{array}{l}
v_{2^{n} k} \rightarrow 0 \\
v_{i} \rightarrow v_{i}, \quad i \neq 2^{n} k
\end{array} \quad, \quad k=0,1,2, \ldots\right.\right.
$$


Notice the relations:

$$
\begin{aligned}
& S(n) P(n)=P(n), \quad P(n) S(n-1)=P(n-1), \\
& P(n-1) P(n)=P(n) P(n-1)=P(n-1) .
\end{aligned}
$$

Let us consider a primary sequence:

$$
(S(1), S(2), \ldots, S(n)) \text {. }
$$

This admits a m-dna sequence $V=a_{1}[S(1)] a_{2}[S(2)] \ldots[S(n)] a_{n+1}$ with $a_{1}=i d$. For example one determines $a_{2}, a_{3}, \ldots$ as:

$$
a_{2}^{*}=S(1), \quad a_{3}^{*}:\left\{\begin{array}{c}
v_{4 k} \rightarrow v_{16 k} \\
v_{4 k+2} \rightarrow v_{2(4 k+2)} \\
v_{2 k+1} \rightarrow v_{2 k+1} \\
(k=0,1, \ldots)
\end{array} \quad, \quad a_{4}^{*}:\left\{\begin{array}{c}
v_{8 k} \rightarrow v_{16(4 k)} \\
v_{8 k+4} \rightarrow v_{4(8 k+4)} \\
v_{4 k+2} \rightarrow v_{2(4 k+2)} \\
v_{2 k+1} \rightarrow v_{2 k+1}
\end{array}, \ldots\right.\right.
$$

Thus $V^{*}=a_{n+1}\left[S(n)^{*}\right] a_{n}\left[S(n-1)^{*}\right] \ldots\left[S(1)^{*}\right] a_{1}$ also consists of a m-dna sequence. Now let us consider a primary sequence:

$$
S=\left(S(n)^{*}, S(n-1)^{*}, \ldots, S(1)^{*}\right) .
$$

Take an ensyme $W=i d[P(n+1)] P(n+1)$ and catalyze it on $V^{*}$ as follows:

$$
\begin{aligned}
V_{1}=V^{*}[W] & =a_{n+1}\left[S(n)^{*} P(n+1)\right] a_{n} P(n)\left[S(n-1)^{*}\right] a_{n-1}\left[S(n-2)^{*}\right] a_{n-2} \ldots \\
& =a_{n+1}[P(n)] a_{n} P(n)\left[S(n-1)^{*}\right] a_{n-1}\left[S(n-2)^{*}\right] a_{n-2} \ldots
\end{aligned}
$$

Then by splicing, $V_{1}$ produces $W_{1}=i d[P(n)] P(n)$. Next let us catalyze $W_{1}$ on $V_{1}$ as follows:

$$
\begin{aligned}
V_{2}=V_{1}\left[W_{1}\right] & =a_{n+1}[P(n)] a_{n} P(n)\left[S(n-1)^{*} P(n)\right] a_{n-1} P(n)\left[S(n-2)^{*}\right] a_{n-2} \ldots \\
& =a_{n+1}[P(n)] a_{n} P(n)[P(n-1)] a_{n-1} P(n)\left[S(n-2)^{*}\right] a_{n-2} \ldots
\end{aligned}
$$

Again by splicing, $V_{2}$ produces $W_{2}=i d[P(n)] P(n)[P(n-1)] P(n-1)$. One can iterate this process until obtaining $W_{n}$. 


\section{Convergence And Divergence}

Let $V$ and $V^{\prime}$ be two infinitely many reflective m-dna sequences. Given a system of mutations $\mathfrak{S}$. Let us denote by $V_{n}=a_{1}(n)\left|X_{1}(n)\right| \ldots a_{l(n)}(n)\left(V_{n}^{\prime}=\right.$ $\left.a_{1}^{\prime}(n)\left|X_{1}^{\prime}(n)\right| \ldots a_{l(n)^{\prime}}(n)\right)$ as $n$-th generations of $V\left(V^{\prime}\right)$ w.r.t. $\mathfrak{S}$. We will say that $V$ and $V^{\prime}$ have convergent evolution w.r.t. $\mathfrak{S}$, if there is $n_{0}$ so that for all $n \geq n_{0}, l(n)=l(n)^{\prime}$ and $X_{j}(n)=X_{j}^{\prime}(n)$ for all $j$.

Let us take two systems of mutations $\mathfrak{S}$ and $\mathfrak{S}^{\prime}$. Let us denote by $V_{n}$ and $V_{n}^{\prime}$ as $n$-th generations of $V$ w.r.t. $\mathfrak{S}$ and $\mathfrak{S}^{\prime}$. We will say that $V$ have divergent evolution w.r.t. $\mathfrak{S}$ and $\mathfrak{S}$, if there is $n_{0}$ so that for all $n \geq n_{0}, l(n) \neq l(n)^{\prime}$ or $l(n)=l(n)^{\prime}$ and $X_{j}(n) \neq X_{j}^{\prime}(n)$ for some $j$.

Example 6.A: Let $H=H_{1} \oplus H_{2} \oplus H_{3}$ where $H_{i}$ are infinite dimensional Hilbert spaces. Let us put the projections on $H_{i}$ by $P_{i}$ and $Q_{i}=i d-P_{i}$. Let us take a m-dna sequence $V=i d\left[Q_{3}\right] Q_{3}\left[Q_{3}\right] i d\left[Q_{2}\right] Q_{2}$. Suppose $V$ accepts two point mutations as:

$$
\begin{aligned}
\nearrow & \nearrow V_{1}=i d\left[Q_{3}\right] Q_{3}\left[P_{1}\right] Q_{2}\left[Q_{2}\right] i d, \\
\searrow V_{2} & =i d\left[Q_{3}\right] Q_{3}\left[Q_{3}\right] i d\left[P_{2}\right] P_{2} .
\end{aligned}
$$

Let us take two ensymes $W_{1}=i d\left|P_{1}\right| P_{1}$ and $W_{2}=i d\left|P_{2}\right| P_{2}$ acting on $V_{i}$ as follows:

$$
\begin{aligned}
& V_{1}\left[W_{1}\right]=i d\left[P_{1}\right] P_{1}\left[P_{1}\right] i d\left[Q_{2}\right] Q_{2}, \quad V_{2}\left[W_{2}\right]=i d\left[Q_{3}\right] Q_{3}\left[P_{2}\right] P_{2}\left[P_{2}\right] i d, \\
& V_{1}\left[W_{2}\right]=i d\left[Q_{3}\right] Q_{3}[0] P_{3}\left[Q_{2}\right] *, \quad V_{2}\left[W_{1}\right]=i d\left[P_{1}\right] P_{1}\left[Q_{3}\right] * .
\end{aligned}
$$

Both of $V_{i}\left[W_{i}\right]$ are m-dna sequences, and both of $V_{1}\left[W_{2}\right]$ and $V_{2}\left[W_{1}\right]$ are not.

\section{Cancering}

Let $V=a_{1}\left|X_{1}\right| \ldots\left|a_{l}\right| X_{l} \mid a_{l+1}$ be a m-dna sequence. A cancering of $V$ at $i$ is a family of state sets:

$$
\left\{\left(a_{1}^{n}, \ldots, a_{l+1}^{n}\right)\right\}_{n}
$$

with the following properties:

(1) each $a_{1}^{n}\left|X_{1}\right| a_{2}^{n}\left|\ldots a_{l}^{n}\right| X_{l} \mid a_{l+1}^{n}$ is a m-dna sequence,

(2) $\operatorname{tr}\left(a_{i}^{n}\left(a_{i}^{n}\right)^{*}\right) \rightarrow+\infty$ 
(3) there is $M>0$ with $\operatorname{tr}\left(a_{j}^{n}\left(a_{j}^{n}\right)^{*}\right) \leq M$ for $i \neq j$.

A m-dna sequence $V$ is stable w.r.t. the mutation system $\mathfrak{S}$, if for any mutation $X_{j} \rightarrow X_{j}^{\prime}$ in $\mathfrak{S}$, the corresponding $V^{\prime}=a_{1}\left|X_{1}\right| \ldots X_{j-1}\left|a_{j}\right| X_{j}^{\prime} \mid a_{j+1} \ldots$ admits also a cancering.

$V$ is unstable, if it is not stable.

Example 7.A: Let $X$ be of finite type (2.F). Then any primary set $\left(X_{1}, \ldots, X_{l}\right)$ admits a cancering if there is a m-dna sequence $V=a_{1}\left|X_{1}\right| a_{2}\left|\ldots a_{l}\right| X_{l} \mid a_{l+1}$ where the state set $\left\{a_{2}, \ldots, a_{l+1}\right\} \subset M_{\infty}(\mathbf{C}),\left(a_{1}=i d\right)$.

Let $\mathfrak{S}$ be consisted by 2.A, 2.B, 2.C,2.E. Then $N$-th stage $\mathfrak{S}^{N}(V)$ can also admit a concering for all $N$.

\section{Metabolism}

Let us take a m-dna sequence $V=a_{1}\left|X_{1}\right| \ldots a_{l}\left|X_{l}\right| a_{l+1}$, and choose an ensyme $W=b_{1}\left|Y_{1}\right| b_{2}\left|\ldots b_{k}\right| Y_{k} \mid b_{k+1}$, where $Y_{j}$ catalyzes on $X_{i}$. Thus there is a state set $\left(c_{1}, \ldots, c_{l+1}\right)$ so that:

$$
V^{\prime}=c_{1}\left|X_{1}\right| \ldots c_{i-1}\left|X_{i-1}\right| c_{i}\left|X_{i} Y_{j}\right| c_{i+1}\left|X_{i+1} \ldots c_{l}\right| X_{l} \mid c_{l+1}
$$

consists of a m-dna sequence. We will denote this catalyzing process as:

$$
V \mapsto V^{\prime}=V[W]
$$

Let us take two m-dna sequences $V$ and $V^{\prime}$. A random metabolism $M$ with input $V$ and output $V^{\prime}$ consists of a family of ensymes $W=\left\{W_{1}, \ldots, W_{l}\right\}$ with the following properties:

(1) one has a diagam of catalizing:

$$
V \rightarrow V_{1}=V\left[W_{1}\right] \rightarrow V_{2}=V_{1}\left[W_{2}\right] \rightarrow \ldots V_{l}=V_{l-1}\left[W_{l}\right]=V^{\prime} .
$$

Definition 8.A: Let $\left\{W_{i}\right\}_{i=1}^{l}$ be a set of ensymes. Then this family is said to be mutually isozyme, if there is another ensyme $w$ and a mutation system $\mathfrak{S}$ so that any elements in $\left\{W_{i}\right\}_{i}$ are obtained by mutation in $\mathfrak{S}$ from $w$.

We will say $w$ is primitive for the family $\left\{W_{i}\right\}_{i}$. 
According to Ureta's hypothesis, enzymes in a metabolism form complexes which are called as isozyme complex.

A constructive metabolism is a random metabolism $M=\left(\left\{V_{i}\right\}_{i},\left\{W_{i}\right\}_{i}\right)$ satisfying the additional properties:

(2) each $W_{i}$ and $W_{j}$ are mutually isozyme,

(3) $W \equiv W_{1} \ldots W_{l}$ is infinitely many reflective.

In the following examples, one will only consider random metabolisms.

8.B Tree-like metabolisms: Let $M_{0}: V_{0} \rightarrow V_{1}$ and $M_{1}: V_{1} \rightarrow V_{2}$ be two metabolisms. Then the composition of these is another:

$$
M_{1} \circ M_{0}: V_{0} \rightarrow V_{1} \rightarrow V_{2} .
$$

Let $T$ be the trivalent graph. Let us equip an orientation on $T$, and let us denote the set of vertices by $v_{0}, v_{1}, v_{2}$, where $v_{0}$ is the input and the others are output ones. For all vertices $v_{i}$, let us assign m-dna sequences $\left\{V, V^{\prime}, V^{1}, V^{2}\right\}$.

A (random) metabolism $M(T)$ with input $V_{0}$ and output $V_{1}$ and $V_{2}$ consists of $\bar{W}^{0}, \bar{W}^{1}$ and $\bar{W}^{2}$ where each $\bar{W}^{i}$ is a family of ensymes $\bar{W}^{i}=\left\{W_{1}^{i}, \ldots, W_{i(l)}^{i}\right\}$ with the following properties:

(1) one has a diagam of catalizing:

$$
\begin{array}{r}
V \rightarrow V_{1}=V\left[W_{1}\right] \rightarrow \ldots V_{l_{0}}=V_{l_{0}-1}\left[W_{l_{0}}\right]=V^{\prime} \\
\searrow V_{1}^{1}=V^{\prime}\left[W_{1}^{1}\right] \ldots V_{l_{1}}^{1}=V^{1}, \\
\searrow V_{1}^{2}=V^{\prime}\left[W_{1}^{2}\right] \ldots V_{l_{2}}^{2}=V^{2} .
\end{array}
$$

When in addition, the following two properties are satisfied, then $M(T)$ will be called constructive:

(2) all $W_{m}^{i}$ and $W_{n}^{j}$ are mutually isozyme.

(3) Let us put $W^{i}=W_{1}^{i} \ldots W_{i(l)}^{i}$. Then $W^{0} \cup W^{1}$ and $W^{0} \cup W^{2}$ are both infinitely many reflective.

By a similar way, one has structure of metabolism $M(T)$ for any finite tree $T$ with a base point. 
8.C Mutant metabolism: Let us have a metabolism $M: V_{0} \mapsto V_{1} \mapsto V_{2}$. Suppose a mutation occurs as $V_{1} \rightarrow V_{1}^{\prime}$ so that $M$ changes to $M^{\prime}$ as follows:

$$
\begin{aligned}
M: V_{0} & \mapsto V_{1} & \mapsto & V_{2} \\
& \searrow & & \nearrow \\
M^{\prime}: & & V_{1}^{\prime} &
\end{aligned}
$$

Then $M^{\prime}: V_{0} \mapsto V_{1}^{\prime} \mapsto V_{2}$ will be called as mutant metabolism.

8.D Reconstruction of metabolisms: Let $V_{0} \rightarrow V_{1}$ and $V_{2} \rightarrow V_{3}$ be metabolisms. Another metabolism $V_{0} \rightarrow V_{3}$ is called a reconstruction of metabolism, if there are no metabolism $V_{1} \rightarrow V_{2}$.

Let us consider a relation with selective splicing. Let $V_{0} \rightarrow V_{1} V_{2}$ and $V_{3} \rightarrow V_{4}$ be metabolisms. Suppose the following:

(1) there is a metabolism $V_{1} \rightarrow V_{3}$, and

(2) there are no metabolism $V_{1} V_{2} \rightarrow V_{3}$.

Then the composit metabolism:

$$
V_{0} \rightarrow V_{1} V_{2}-\text { selective splicing } \rightarrow V_{1} \rightarrow V_{3} \rightarrow V_{4}
$$

is said to be an extended metabolism by self splicing.

8.E Connection by succesive self splicing: Let $M_{0}: U_{0} \rightarrow U_{1}$ and $M_{1}$ : $U_{2} \rightarrow U_{3}$ be two metabolisms. Let us take an ensyme $W$ catalyzing on $U_{1}$, $V_{2}=U_{1}[W]$. Then it produces $W_{2}$ by splicing. Next let $W_{2}$ catalyze on $V_{2}$, $V_{3}=V_{2}\left[W_{2}\right]$. Then again it produces $W_{3}$ by splicing. On may iterate this process, and finally obtain $W_{l}$. If $W_{l}$ catalyzes on $U_{2}$ which switches on the metabolic system $M_{1}$, then this connecting process of $M_{0}$ with $M_{1}$ will be called as a connection by succesive self splicing (cf. 5.C). Here is a diagram:

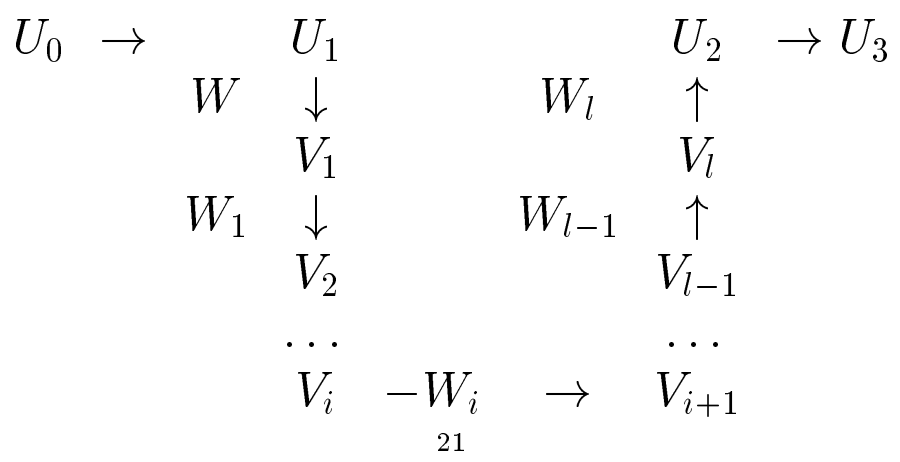


When there are two $W$ and $W^{\prime}$ such that $W_{1}=W W^{\prime}, W_{2}=W W^{\prime} W, W_{3}=$ $W W^{\prime} W W^{\prime}=\left(W W^{\prime}\right)^{2}, W_{4}=\left(W W^{\prime}\right)^{2} W, \ldots$, then the process will be called as a cyclic connection.

8.F Join of metabolisms: A particular case of connection is called join. Let us take a metabolism system:

$$
M_{1}: V_{0} \mapsto V_{1} V_{2} \mapsto V_{3} \mapsto \ldots, \quad M_{2}: U_{0} \mapsto U_{1} \mapsto \ldots
$$

Suppose the followings:

(1) an ensyme $W$ catalyzes on $V_{1} V_{2}$ so that the self splicing $V_{1} V_{2} \mapsto V_{1}$ occurs,

(2) $V_{1}$ catalyzes on $U_{0}$ with $U_{0}\left[V_{1}\right]=U_{1}$.

Then the following metabolism is called join of $M_{1}$ with $M_{2}$ :

$$
\begin{aligned}
V_{0} \mapsto V_{1} V_{2} \mapsto \quad & V_{1} \\
& \downarrow \\
& U_{0} \mapsto U_{1} \mapsto U_{2} \mapsto \ldots
\end{aligned}
$$

Example 8.F: Let us consider a case when $M_{1}$ and $M_{2}$ are metabolisms consisted by projection states and shift states respectively. Let us choose:

$$
S\left\{\begin{array}{l}
v_{2 i} \mapsto v_{2 i+2} \\
v_{2 i+1} \mapsto v_{2 i+1}
\end{array} \quad, \quad P_{1}\left\{\begin{array}{l}
v_{0} \mapsto v_{0} \\
v_{2 i} \mapsto 0, \quad i \geq 1 \\
v_{2 i+1} \mapsto v_{2 i+1}
\end{array} \quad, \quad P_{2}\left\{\begin{array}{l}
v_{2 i} \mapsto 0 \\
v_{2 i+1} \mapsto v_{2 i+1}
\end{array}\right.\right.\right.
$$

Let us take a m-dna sequence $V$ and consider a metabolic system:

$$
\begin{aligned}
& M_{1}: V_{0} \mapsto i d[S] S V \equiv V_{1} V \mapsto V_{2} \ldots \text { (shift system), } \\
& M_{2}: U_{0} \mapsto i d\left[P_{1}\right] P_{1} U \equiv U_{1} \mapsto i d\left[P_{2}\right] P_{2} U \mapsto \ldots \text { (projective system). }
\end{aligned}
$$

Since $P_{1} S=P_{2}$, one may catalyze as $i d\left[P_{1}\right] P_{1}(i d[S] S)=i d\left[P_{2}\right] P_{2}$. Thus one can make join of $M_{1}$ with $M_{2}$.

8.G Maximal connecting metabolism: Let us take two m-dna sequences $V_{0}$ and $V_{1}$, and consider two metabolisms:

$$
M_{1}: V_{0} \rightarrow U_{1} \rightarrow U_{2} \rightarrow \ldots U_{l} \rightarrow V_{1}, \quad M_{22}: V_{0} \rightarrow U_{1}^{\prime} \rightarrow U_{2}^{\prime} \rightarrow \ldots U_{k}^{\prime} \rightarrow V_{1} .
$$


Then if $i$ is the maximal number satisfying that there is a metabolism $M: U_{i} \rightarrow$ $U_{i}^{\prime}$, then $M$ is called a maximal connecting metabolism:

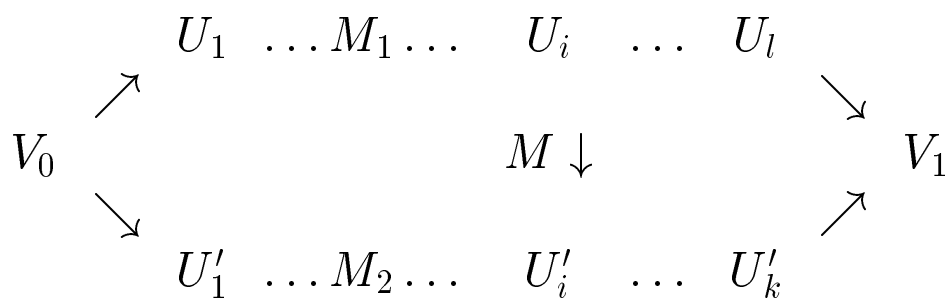

Example 8.G: Let $S(n)$ and $P(n)$ be in the example 5.C:

$$
S(n):\left\{\begin{array}{l}
v_{2^{n} k} \rightarrow v_{2^{n+1} k} \\
v_{i} \rightarrow v_{i}, \quad i \neq 2^{n} k
\end{array} \quad, \quad P(n):\left\{\begin{array}{l}
v_{2^{n} k} \rightarrow 0 \\
v_{i} \rightarrow v_{i}, \quad i \neq 2^{n} k
\end{array}\right.\right.
$$

Let us choose two metabolisms $M_{1}$ and $M_{2}$, where $M_{1}$ is a shift system and $M_{2}$ is a projective system as:

$$
\begin{aligned}
& M_{1}: i d[i d] i d \mapsto i d[S(3 m)] S(3 m)^{*} \mapsto i d[S(3 m-2)] S(3 m-2)^{*} \mapsto \ldots \\
& \mapsto i d[S(3 m-2 j)] S(3 m-2 j)^{*} \mapsto \ldots \\
& \mapsto i d[S(m)] S^{m} \mapsto i d[S(m-2)] S(m-2)^{*} \ldots \\
& \quad \mapsto i d[S(1)] S(1)^{*} \mapsto i d[P(1)] P(1), \\
& M_{2}: i d[i d] i d \mapsto i d[P(2 m)] P(2 m) \mapsto i d[P(2 m-1)] P(2 m-1) \mapsto \ldots \\
& \mapsto i d[P(2 m-j)] P(2 m-j) \mapsto \ldots \\
& \mapsto i d[P(m)] P(m) \mapsto i d[P(m-1)] P(m-1) \cdots \mapsto i d[P(1)] P(1) .
\end{aligned}
$$

Notice that there is a metabolism from $i d[S(i)] S(i)^{*}$ to $i d[P(j)] P(j)$ if and only if $i \geq j$. Thus the maximal connecting metabolism is

$$
M: i d[S(m)] S(m)_{23}^{*} \mapsto i d[P(m)] P(m)
$$


8.H Convergence of metabolisms: Let us take two m-dna sequences $V_{0}$ and $V_{1}$. Suppose there are two metabolisms:

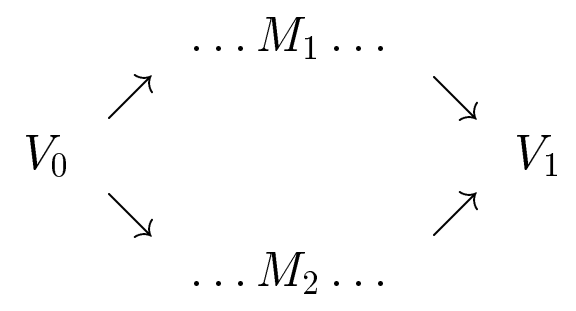

Let us take two m-dna sequences $V^{\prime}$ and $V^{\prime \prime}, V^{\prime}, V^{\prime \prime} \neq V_{0}, V_{1}$. Suppose there exists another metabolism $M_{3}: V^{\prime} \rightarrow V^{\prime \prime}$ :

$$
\begin{gathered}
M_{1}: V_{0} \rightarrow V^{\prime} \rightarrow V_{1} \\
M_{3} \downarrow \\
M_{2}: V_{0} \rightarrow V^{\prime \prime} \rightarrow V_{1} .
\end{gathered}
$$

Then we will say that $M_{1}$ and $M_{2}$ are connected. If $M_{1}$ and $M_{2}$ are non connected, then we will say that these metabolisms are convergent systems.

Example 8.H: Let us take two operators $X_{1}, X_{2}$ such that there are no $A$ and $A^{\prime}$ with $X_{1} A=X_{2}$ and $X_{2} A^{\prime}=X_{1}$. If one can choose $V^{\prime}=i d\left[X_{1}\right] X_{1}^{*}$ and $V^{\prime \prime}=i d\left[X_{2}\right] X_{2}^{*}$, then $M_{1}$ and $M_{2}$ are convergent systems.

Let $P_{0}, P_{1}, P_{2}, P_{3}, P_{4}$ be projections satisfying:

$$
P_{0} P_{3}=P_{3}, \quad P_{1} P_{3}=P_{1}, \quad P_{1} P_{2}=P_{2}, \quad P_{2} P_{4}=P_{4} .
$$

Let us consider metabolisms:

$$
\begin{aligned}
& M_{1}: V_{0}=i d\left[P_{0}\right] P_{0}\left[P_{2}\right] P_{2} \mapsto V^{\prime}=i d\left[P_{1}\right] P_{1}\left[P_{2}\right] P_{2} \mapsto V_{1}=i d\left[P_{1}\right] P_{1}\left[P_{4}\right] P_{4}, \\
& M_{2}: V_{0}=i d\left[P_{0}\right] P_{0}\left[P_{2}\right] P_{2} \mapsto V^{\prime \prime}=i d\left[P_{0}\right] P_{0}\left[P_{4}\right] P_{4} \mapsto V_{1}=i d\left[P_{1}\right] P_{1}\left[P_{4}\right] P_{4} .
\end{aligned}
$$

Then there are no metabolism as $V^{\prime} \rightarrow V^{\prime \prime}$.

In practice there are some pairs $\left(W, W^{\prime}\right)$ of ensymes such that $W$ and $W^{\prime}$ play the same role as functions, however many of structures of genes (from primary to fourth) are very different mutually. Such pair is said to be obtained by convergent evolution. For example kimo-tripsin and subtilisin consists such pair. 
8.I Divergence of metabolisms: Let us consider two metabolisms $M_{1}: V_{0} \rightarrow$ $V^{\prime}$ and $M_{2}: V_{0} \rightarrow V^{\prime \prime}:$

$$
V_{0} \rightarrow V_{1} \underset{M_{2}}{\stackrel{M_{1}}{\rightarrow}} \nearrow{ }^{V^{\prime \prime}}
$$

If there are no invertible metabolisms as below:

$$
V^{\prime} \rightarrow V^{\prime \prime}, \quad V^{\prime \prime} \rightarrow V^{\prime}
$$

then we will say that $M_{1}$ and $M_{2}$ are divergent systems.

Example 8.I: Let $S(n)$ and $P(n)$ be in the example 5.C. Let us consider two metabolisms:

$$
\begin{aligned}
& M_{1}: i d[S(n+2)] S(n+2)^{*} \mapsto i d[S(n+1)] S(n+1)^{*} \mapsto i d[S(n)] S(n)^{*}, \\
& M_{2}: i d[P(n+2)] P(n+2) \mapsto i d[P(n+1)] P(n+1) \mapsto i d[P(n)] P(n) .
\end{aligned}
$$

The ensyme $i d[P(n)] P(n)$ can catalyze on $i d[S(n)] S(n)$ which produces a metabolism $i d[S(n)] S(n)^{*} \mapsto i d[P(n)] P(n)$. However there are no metabolism from $i d[P(n)] P(n)$ to $i d[S(n)] S(n)^{*}$.

In practice a pair of two ensymes is said to be obtained by divergent evolution if they arose from the same original one, and have been developped very differently.

8.J Cyclic metabolism: Let us take m-dna sequences $V, V^{\prime}$ such that both of $V V^{\prime}$ and $V^{\prime} V$ are also m-dna sequences. Suppose there is a family of metabolisms $\left\{M_{n}\right\}_{n \geq 0}$ :

$$
\begin{aligned}
& M_{0}: V \rightarrow V V^{\prime}, \quad M_{1}: V V^{\prime} \mapsto V V^{\prime} V, \\
& M_{2}: V V^{\prime} V \mapsto V V^{\prime} V V^{\prime}, \quad M_{3}: V V^{\prime} V V^{\prime} \mapsto V V^{\prime} V V^{\prime} V, \quad \ldots
\end{aligned}
$$

Thus inductively $M_{2 n} \circ \cdots \circ M_{0}$ produces $\left(V V^{\prime}\right)^{n} \equiv V V^{\prime} \ldots V V^{\prime}$ and $M_{2 n+1} \circ$ $\cdots \circ M_{0}$ produces $\left(V V^{\prime}\right)^{n} V$ as metabolism productions. We will say that the family $\left\{M_{n}, W_{n}\right\}_{n}$ is a cyclic metabolism system, where $W_{n}$ are ensymes for $M_{n}$. 
We will denote pictorically as:

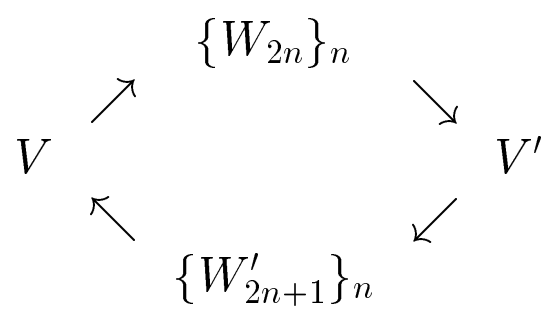

We will say that the metabolic system $M=\left\{M_{i}\right\}_{i}$ is a cyclic metabolism from $V$ to $V^{\prime \prime}=\left(V V^{\prime}\right)^{n}$ (or to $\left.\left(V V^{\prime}\right)^{n} V\right)$.

Example 8.J: Here we have three types of metabolic systems:

(1) Let us take a long m-dna sequence $\tilde{V}$ which contains many copies of $V$ and $V^{\prime}$. When there is a family of ensymes $\left\{W_{n}\right\}_{n}$ acting on $\tilde{V}$ such that for each $n, W_{n}$ catalyzes on $\tilde{V}$ which helps it to make self splicing.

(2) Let $\tilde{V}$ be as above. Suppose there is an ensyme $W$ which catalyzes on $\tilde{V}$ and they produce by self splicing, $V V^{\prime}$. Next suppose $W_{2} \equiv V V^{\prime}$ catalyzes on $\tilde{V}$ and they produce by self splicing, $V V^{\prime} V$. Succesively $V V^{\prime} V$ catalyzes on $\tilde{V}$ producing $V V^{\prime} V V^{\prime}$ by self splicing. One may iterate this process. This metabolism will be called as a self production of cyclic metabolism.

(3) Periodic inequally crossing over.

8.K Minimal metabolism for extension: Let us take a metabolism family:

$$
M_{0}: V_{0} \rightarrow V_{1}, \quad M_{1}: V_{1} \rightarrow V_{2}, \ldots, M_{n}: V_{n} \rightarrow V_{n+1}, \ldots
$$

Let us choose a particular m-dna sequence $U$. Then if $i$ is the minimal number satisfying that there is a metabolism $M: V_{0} \rightarrow U \rightarrow V_{i+1}$, then we will say that the composition $M_{i} \circ \cdots \circ M_{0}$ is called a minimal metabolism for extension w.r.t. $\left(M_{i} ; U\right)$ and $i$ is the minimal number of extension:

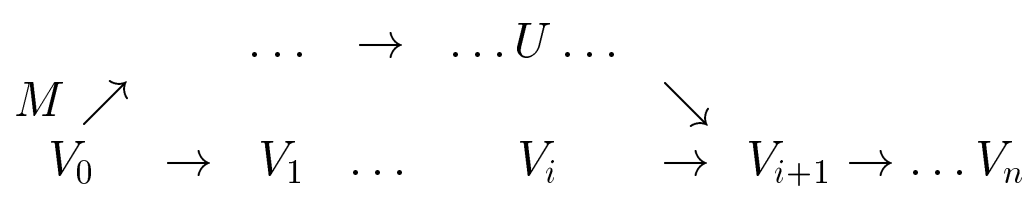

Example 8.K: Let $S(m)$ and $P(m)$ be in 5.C and choose $U=i d[S(m)] S(m)^{*}$, 
$m \leq n$. Let us choose metabolisms:

$$
\begin{aligned}
& M_{0}: V_{0}=i d[i d] i d \mapsto V_{1}=i d[P(n)] P(n), \\
& M_{l}: V_{l}=i d[P(n-l+1)] P(n-l+1) \mapsto V_{l+1}, \quad 1 \leq l \leq n .
\end{aligned}
$$

Let us find $M: i d[i d] i d \mapsto i d[S(m)] S(m)^{*} \mapsto i d\left[P\left(m^{\prime}\right)\right] P\left(m^{\prime}\right)$. Then $M$ exists only when $m^{\prime} \leq m$. Thus the minimal number of extension is $n-m$.

8.L Locally amplification of metabolic systems: Let us consider a family of metabolisms $M_{0}: V_{0} \rightarrow V_{1}, \quad M_{1}: V_{1} \rightarrow V_{2}, \ldots$ If there is another family of metabolisms $M: V_{i} \rightarrow U_{1} \ldots U_{l} \rightarrow V_{i+1}$, then the following extended system of metabolisms will be called as a locally amplification of metabolic systems:

$$
\begin{array}{rllll}
V_{0} \rightarrow \ldots & V_{i} & \rightarrow & V_{i+1} & \rightarrow \ldots \\
& \downarrow & & \uparrow & \\
& U_{1} & & U_{l} & \\
& \downarrow & M & \uparrow \\
& U_{2} & & U_{l-1} \\
& & & \\
& & & \\
& U_{i} & \rightarrow & U_{i+1}
\end{array}
$$

If $M$ is a cyclic metabolism from $V_{i}$ to $V_{i+1}$, then we will say that the above system will be called as a locally cyclic amplification of metabolic systems.

Example 8.L: Let us take another m-dna sequences $V_{i}^{\prime}$ and $V_{i+1}^{\prime}$ so that there is a cyclic metabolism $V_{i}^{\prime} \rightarrow V_{i+1}^{\prime}$. Now suppose a mutation occurs $V_{i} \rightarrow V_{i}^{\prime}$ so that a mutant metabolism $M^{\prime}$ is produced:

$$
\begin{array}{rlrlll}
M: V_{0} \mapsto \ldots V_{i-1} & \mapsto V_{i} & \mapsto V_{i+1} \mapsto \ldots \\
M^{\prime}: & & \searrow & & \nearrow \\
V_{i}^{\prime} & &
\end{array}
$$

If $M^{\prime}$ makes locally cyclic amplification of metabolic systems as below, then it will be called as locally cyclic amplification of mutant metabolic systems:

$$
\begin{array}{rlll}
V_{i+1} & \leftarrow & \ldots & \quad\left(V_{i}^{\prime} V_{i+1}^{\prime}\right)^{l} \quad \ldots \\
\uparrow & \nwarrow & \\
V_{i-1} & \mapsto V_{i}^{\prime} \mapsto V_{i}^{\prime} V_{i+1}^{\prime} \mapsto \ldots
\end{array}
$$


8.M Enlargement: Let $M_{0}: V_{0} \rightarrow V_{1} \rightarrow V_{2} \rightarrow \ldots$ and $M_{1}: V_{0}^{1} \rightarrow V_{1}^{1} \rightarrow \ldots$ be systems of metabolisms. Let us choose $i$. An enlargement of $M_{0}$ and $M_{1}$ at $i$ is catalyzing of $V_{i}^{1}$ on $V_{i}$ as an enzyme.

Suppose one has a family of metabolic systems $\left\{M_{i}: V_{0}^{i} \rightarrow V_{1}^{i} \rightarrow \ldots\right\}_{i}$. An enlargement of the family at $i$, is succesively catalyzing of $V_{i}^{l}$ on $V_{i}^{i+1}$ as an ensyme. Thus one has a diagram:

$$
\begin{aligned}
& M_{0}: V_{0} \rightarrow V_{1} \rightarrow V_{2} \quad \rightarrow \quad \ldots \quad \rightarrow \quad V_{i} \rightarrow V_{i+1} \ldots \\
& M_{1}: V_{0}^{1} \rightarrow V_{1}^{1} \ldots \quad \rightarrow \quad V_{i}^{1} \rightarrow V_{i+1}^{1} \quad \ldots \\
& M_{2}: V_{0}^{2} \rightarrow V_{1}^{2} \cdots \rightarrow V_{i}^{2} \rightarrow V_{i+1}^{2} \cdots
\end{aligned}
$$

8.N Branching of a metabolism: Let us consider a metabolism $M: V_{0} \mapsto$ $V_{1} \mapsto V_{2} \ldots$ Suppose $V_{1}$ catalyzes on $V_{0}$ which produces another $U_{1}$. Then one gets the diagram:

$$
M: V_{0} \longmapsto V_{1} \mapsto V_{2} \ldots
$$

Succesively let $V_{2}$ calatyze on $U_{1}$ which produces $U_{2}$. Inductively one considers $U_{i}$ which is the production of $V_{i}$ catalyzing on $U_{i-1}$ :

$$
\begin{aligned}
& V_{0} \mapsto V_{1} \quad \mapsto V_{2} \ldots \\
& U_{1} \mapsto U_{2} \mapsto U_{3} \ldots
\end{aligned}
$$

Then we will say that the above new metabolism is the branched metabolism.

8.O Reconstruction of metabolic systems: Let:

$$
\bar{M}=\left\{\left(M_{1}, \ldots, M_{l}\right),\left(\left\{V_{i, j}\right\}_{i, j \in I}\right)\right\}
$$

be a metabolic system where $V_{i, j}$ is a join of $M_{i}$ with $M_{j}$. If there is another system $\bar{M}^{\prime}=\left\{\left(M_{1}, \ldots, M_{l}\right),\left(\left\{V_{i, j}^{\prime}\right\}_{i, j \in I}\right)\right\}$, then we will say that $\bar{M}^{\prime}$ is a reconstruction of $\bar{M}$. 
8.P Homeobox: Let $P$ be a $\mathrm{m}$-dna sequence which plays a role of a promotor. Let us take another family of m-dna sequences $\left\{V_{0}, V_{1}, \ldots, V_{l}\right\}$ where all $P V_{j}$ are also m-dna sequences.

Suppose there is a metabolism:

$$
M: P V_{0} \rightarrow P V_{1} \rightarrow \ldots P V_{l} .
$$

If there is a mutation $P \rightarrow P^{\prime}$ which consists of another metabolism:

$$
M^{\prime}: P^{\prime} V_{i_{0}} \rightarrow P^{\prime} V_{i_{1}} \rightarrow \ldots P^{\prime} V_{i_{l}}
$$

then we will say that $P$ is a homeobox.

Let us take $i<j$. If $M^{\prime}$ is of the form below, then the mutation will be called simple :

$$
\begin{aligned}
& M: P V_{0} \rightarrow \ldots P V_{i-1} \rightarrow P V_{i} \rightarrow P V_{i+1} \rightarrow \quad \ldots \\
& M^{\prime}: P^{\prime} V_{0} \rightarrow \ldots P^{\prime} V_{i-1} \rightarrow \underset{\mathbb{1}}{P^{\prime} V_{j}} \rightarrow P^{\prime} V_{i+1} \rightarrow \quad \ldots \\
& \ldots \quad P V_{j-1} \rightarrow \quad P V_{j} \quad \rightarrow P V_{j+1} \ldots
\end{aligned}
$$

8.Q Evolution of metabolisms: Let $M$ be a metabolism. When $M$ makes development under a mutation system $\mathfrak{S}$, then one will get a family of metabolisms. This will be called as a evolution of $M$ under $\mathfrak{S}$.

\section{Conservative System in Metabolisms}

Here one introduces an assumption on the conservation of the total production by metabolisms. Let $\bar{M}=\left\{M_{1}, \ldots, M_{n}\right\}$ be a system of metabolisms. Suppose a mutation occurs on this system, and it changes into $\bar{M}^{\prime}=\left\{M_{1}^{\prime}, \ldots, M_{n^{\prime}}^{\prime}\right\}$. Then the assumption saids that if $\bar{M}^{\prime}$ produces new m-dna sequences, then it should be consumed as ensymes inside $\bar{M}^{\prime}$.

Let us consider by an example. Suppose a situation; a metabolic system makes an amplification by mutation which produces a new ensyme $W^{\prime}$. Then 
it can be consumed to make a cyclic system. Let us take a cyclic system:

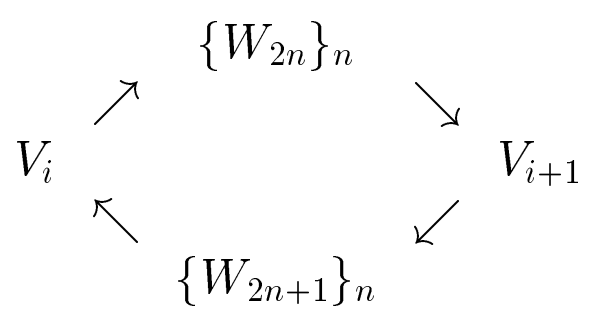

Then we consider a metabolic system $\bar{M}=\left\{M_{1}, M_{2}\right\}$ :

$$
M_{0}: V_{0} \mapsto \ldots V_{i} \mapsto V_{i+1} \mapsto \ldots V_{n}, \quad M_{1}: U \mapsto \ldots U^{\prime}
$$

Suppose a connection between $M_{0}$ and $M_{1}$ occurs as $V_{n} \mapsto U$, by a help of an ensyme $W$. Suppose $U^{\prime}$ catalyzes on $V_{i}$ which makes a loc. amp. of metabolism on $V_{i} \mapsto V_{i+1}$. Thus one will have the following diagram:

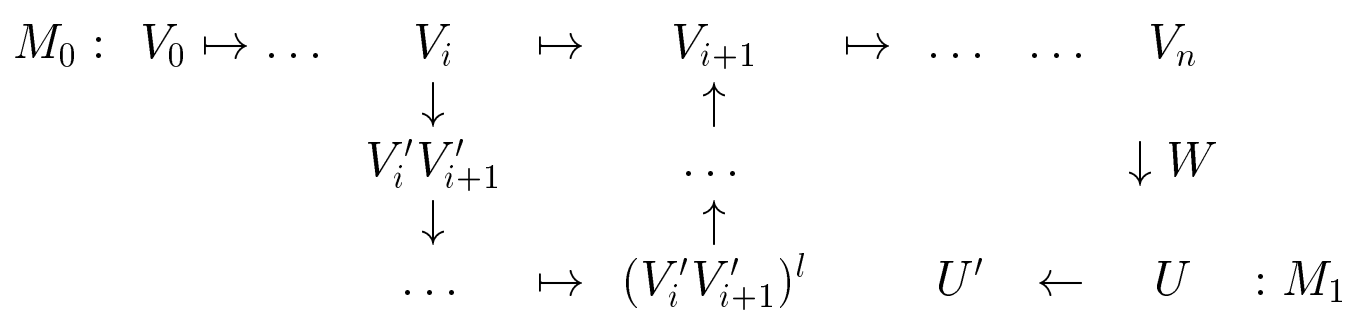

Thus in order to consume $U^{\prime}$, this metabolic system has produced a locally amplification.

\section{Glycolysis}

In this section, one will make a partial construction of a metabolic system, G-system, modelled on a glycolysis (Embden-Meyerhof-Parnas pathway). Let us take two m-dna sequences $G c$ and $P_{2}$, and two ensymes $P K$ and $P P$. Then G-system is a metabolic system beginning from $G c$ and ending by producing $P_{2}$ where one uses $P K, P P$ and their variations as ensyme families.

There are several steps and here we will concentrate on the following steps:

(1) $G c$ (glucose) $\rightarrow G l$ (glycogen),

(2) $F_{1}$ (fructose-6-phosphate) $\rightarrow F_{3}$ (fructose-2,6-2-phosphate),

(3) $P_{1}$ (phospho-enol-pilbin) $\rightarrow P_{2}$ (pilbin).

During the above steps, several ensymes are interacting mutually. At every 
step, we will use two ensymes, $P K$ (proteinkinase) and $P P$ (phospho-proteinphosphatase). These have some different activation states respectively.

10.A From glucose to glycogen: Let us begin from (1). An ensyme $P$ (phospholirase) activates on $G c$ and produces $G l$ :

$$
G c-[P] \longrightarrow G l \text {. }
$$

$P$ has three, from high to low (in reality four) activation states:

$$
\text { (high) } P \longleftrightarrow P^{\prime \prime} \longleftrightarrow P^{\prime} \text { (low) }
$$

As ensymes, $P P, P K$ and $G l$ act on $P$ as the diagram below:

$$
P^{\prime \prime} \begin{array}{ccccc}
{[P P] \nearrow} & & P^{\prime} & & \\
\leftarrow & {[G l]} & - & & \\
& {[P K]} &
\end{array}
$$

Thus $P K$ makes $P$ active, and $G l$ do it actless.

In fact the metabolism $G c \rightarrow G l$ is inversible. There is another ensyme $S t$ (glycogensyntase) catalyzing as below:

$$
\begin{aligned}
& G c \stackrel{[P]}{\longleftrightarrow} \overrightarrow{G l} \\
& \leftarrow[S t]
\end{aligned}
$$

$S t$ has also three activating states; $S t$ and $S t^{\prime \prime}$ are both active, and $S^{\prime}$ is actless:

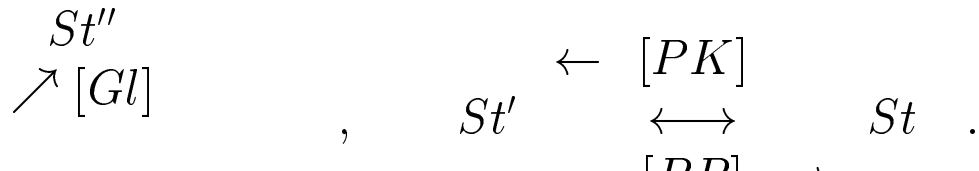

$$
\begin{aligned}
& S t^{\prime} \longleftrightarrow S t \\
& {[P P] \rightarrow}
\end{aligned}
$$

Example 10.A: Let $H$ be an infinite dimensional Hilbert space, and take basis $\left\{\ldots, v_{-l}, \ldots, v_{0}, v_{1}, \ldots\right\}$. Let $S$ be a shift, $S: v_{i} \rightarrow v_{i+1}$. $S$ is invertible. Let us begin with:

$$
G c=i d\left[S^{*}\right] S \mapsto G l=i d[i d] i d, \quad P P=i d[S] S^{*}, P K=i d\left[S^{*}\right] S .
$$


Then one deteremines $P$ and $S t$ as:

$$
\begin{aligned}
& P=i d[S] S^{*}, \quad P^{\prime}=S\left[S^{2}\right] S^{*}, \quad P^{\prime \prime}=S[S] i d, \\
& S t=i d\left[S^{*}\right] S, \quad S t^{\prime}=S t^{\prime \prime}=i d\left[S^{2 *}\right] S^{2} .
\end{aligned}
$$

Let us put this metabolism by $M_{1}(S)^{\prime}$. In this example, one may replace $S$ by $S^{*}=S^{-1}$. Thus $M_{1}\left(S^{*}\right)^{\prime}$ gives another one. Let us put $H^{\prime}=H \oplus H$. Then by identifying $H^{\prime}$ with $H$, the direct sum $T=S \oplus S^{*}$ also gives a metabolism. The direct sum of metabolisms will be expressed as:

$$
M_{1}(S)^{\prime} \oplus M_{1}\left(S^{*}\right)^{\prime}=M_{1}\left(S \oplus S^{*}\right)^{\prime} .
$$

10.B Activating states on PP: There is an ensyme $P I$ (phosphatase-inhibitar) which makes $P P$ actless. This also has two states, high and low:

$$
\text { (high) } P P, P I \longleftrightarrow P P^{\prime}, P I^{\prime} \text { (low) . }
$$

These are mutually interacting and have the following metabolic systems:

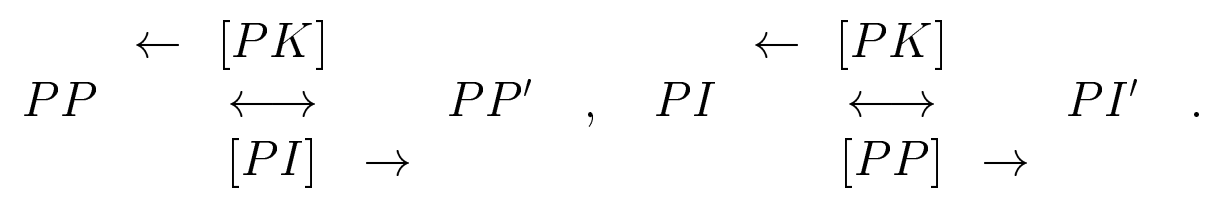

Let us consider the above metabolic system. It expresses states of $P P$ and $P I$ catalyzed by ensymes $P K$ and $P P$. Combinig with these, one has a diagram:

$$
\begin{aligned}
& P P \stackrel{P K}{\underset{P I}{\longleftrightarrow} \rightarrow} \rightarrow P^{\prime} \\
& P P \quad \underset{ }{\downarrow \uparrow} \quad P K \\
& P I^{\prime}
\end{aligned}
$$

Let us observe the following; for example, suppose this system begins with $P P^{\prime}$ and $P I^{\prime}$, and supply $P K$ as an ensyme. Then the states change into $P P$ and $P I$. Then $P P$ changes into $P P^{\prime}$ catalyzed by $P I$. Also $P I$ changes into $P I^{\prime}$ catalyzed by $P P$. Thus it comes back to the intial state in this system:

$$
\left.P K:\left(P P^{\prime}, P I^{\prime}\right) \rightarrow \underset{32}{(P P}, P I\right) \rightarrow\left(P P^{\prime}, P I^{\prime}\right) .
$$


When one changes the initial states as $\left(P P\left({ }^{\prime}\right), P I\left({ }^{\prime}\right)\right)$, then there are similar occurence.

Definition 10.B: Let us consider a metabolic system $M$. If an ensyme $W(=$ $P K)$ satisfies the above property, then it is called stable.

Example 10.B: Let us continue the above example. The following extension gives an example of metabolism from glucose to glycogen:

$$
P I=S[S] i d, \quad P I^{\prime}=S\left[S^{2}\right] S^{*}, \quad P P^{\prime}=S\left[S^{2}\right] S^{*} .
$$

We will denote this as $M_{1}(S)$.

Again one may also replace $S$ by $T=S \oplus S^{*}$, and get $M_{1}(T)=M_{1}\left(S \oplus S^{*}\right)$.

10.C Fluctose metabolic system: There are several ensymes:

$$
\begin{array}{ll}
P F K_{i} & \text { (phospho-fluctokinase), } \\
F B P_{i} & \text { (fluctose-bisphosphatase), }
\end{array}
$$

$i=1,2$ with the diagram:

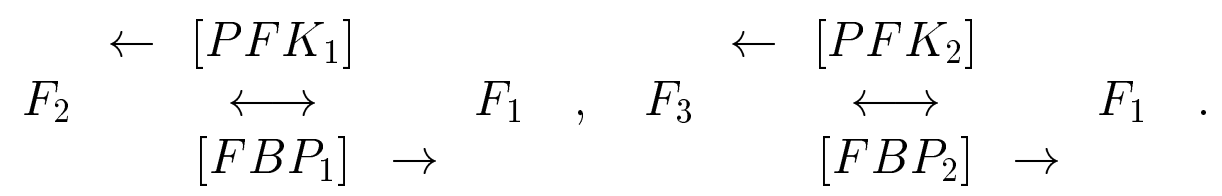

Both of $P F K_{i}$ and $F B P_{i}$ have high and low states mutually. We will denote low state by adding dash, $P F K_{i}^{\prime}$ and $F B P_{i}^{\prime}$. The fluctose metabolic system has a mutual interaction as:

$$
\begin{aligned}
& \text { (high) } P F K_{1} \leftarrow \underset{[C]}{\stackrel{\left[F_{1}\right],\left[F_{3}\right]}{\longleftrightarrow}} \rightarrow \text { PFK (low) } \\
& \text { (high) FBP } \stackrel{\leftarrow}{\overleftrightarrow{\left[F_{3}\right]} \rightarrow} \rightarrow F B P_{1}^{\prime} \text { (low) }
\end{aligned}
$$

where $C$ is Cuen-acid which comes from the Cuen cycle after glucose one. Here we will not take into account of the effect of $C$.

10.D Some properties of $P F K$ and $F B P$ : $P F K_{2}$ and $F B P_{2}$ have isozymes 
respectively, and in order to distinguish them, we will express the isozyme pairs by:

$$
\left(\mathrm{LPFK}_{2}, \mathrm{HPF} \mathrm{K}_{2}\right), \quad\left(\mathrm{LFBP}_{2}, \mathrm{HFBP}_{2}\right) .
$$

$P K$ and $P P$ catalyze on both of $L$ and $H$ types which are inverse way as:

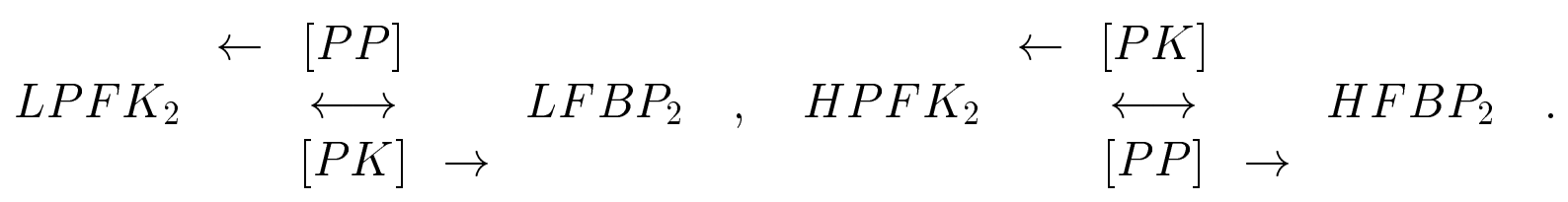

Let us take two m-dna sequences $V, V^{\prime}$ and an ensyme $S$ catalyzing as below:

$$
\begin{array}{ll}
V \\
\downarrow \\
\downarrow \uparrow \\
V^{\prime}
\end{array}
$$

The above type of $S$ will be called a partial involution.

Let us take pairs of isozymes $(V, U)$ and $\left(V^{\prime}, U^{\prime}\right)$. Suppose an ensyme $S$ catalyzes as below:

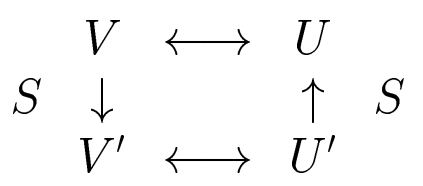

Then this type of $S$ will be called as quasi partial involution.

\section{Example 10.D:}

(1) Involution: Let us decompose $H=H_{1} \oplus H_{2}$, where $H_{1}=\left\{v_{2 i+1}\right\}_{i}$ and $H_{2}=\left\{v_{2 i}\right\}_{i}$. Then one puts:

$$
I_{0}: v_{2 i} \rightarrow v_{2 i+1}, \quad v_{2 i+1} \rightarrow v_{2 i}
$$

$I_{0}$ is an involution.

(2) Partial involution: $H$ has another splitting $H=H_{1}^{\prime} \oplus H_{2}^{\prime} \oplus H_{3}^{\prime}$ where $H_{1}^{\prime}$ is spanned by $v_{4 i}, H_{2}^{\prime}$ by $v_{4 i+2}$ and $H_{3}^{\prime}$ by $v_{2 i+1}$. Then by a similar way, there is an involution $I_{0}^{\prime}: H_{1}^{\prime} \cong H_{2}^{\prime}$. Let us put:

$$
I\left|H_{1}^{\prime} \oplus H_{2}^{\prime} \equiv I_{0}^{\prime}, \quad I\right| H_{3}^{\prime} \equiv 0 .
$$

Then $I$ is a partial involution. 
(3) Quasi partial involution: Let $I_{0}$ be as above, and define another shift $T$ as:

$$
T: v_{2 i} \rightarrow v_{2 i+2}, \quad v_{2 i+1} \rightarrow v_{2 i-1} .
$$

Then one has the following commutative diagram:

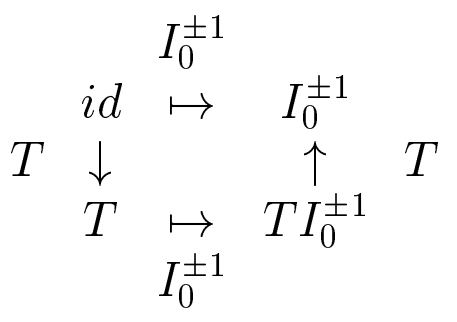

$I_{0}$ is a quasi partial involution between $\left(i d, I_{0}^{ \pm 1}\right)$ and $\left(T, T I_{0}^{ \pm 1}\right)$.

Now using the above example, one gets an example of fluctose metabolic system below. It will be an extension of $M_{1}(T)$. Let us choose:

$$
\begin{aligned}
& P P=i d[T] T^{*}, \quad P K=i d\left[T^{*}\right] T, \\
& \left(L F B P_{2}, H F B P_{2}\right)=\left(i d[i d] i d, i d\left[I_{0}\right] I_{0}^{*}\right), \\
& \left(L P F K_{2}, H P F K_{2}\right)=\left(i d[T] T^{*}, i d\left[T I_{0}\right] I_{0}^{*} T^{*}\right) .
\end{aligned}
$$

This extended example of fluctose metabolism will be denoted as $M_{2}\left(T, I_{0}\right)^{\prime}$.

Let $S$ be the shift $v_{i} \rightarrow v_{i+1}$ as before, and have a metabolism $S^{*}\left(M_{2}\left(T, I_{0}\right)^{\prime}\right)$ by:

$$
\begin{aligned}
& P P=i d[T] T^{*}, \quad P K=i d\left[T^{*}\right] T, \\
& \left(L F B P_{2}, H F B P_{2}\right)=\left(i d[S] S^{*}, i d\left[S I_{0}\right] I_{0}^{*} S^{*}\right), \\
& \left(L P F K_{2}, H P F K_{2}\right)=\left(i d[S T] T^{*}, i d\left[S T I_{0}\right] I_{0}^{*} T^{*} S^{*}\right) .
\end{aligned}
$$

Let us consider interaction between $F_{1}$ and $F_{3}$ above. Using the above example, one will obtain an example of $F_{1}$ and $F_{3}$ catalyzed by $\left(L F B P_{2}, L P F K_{2}\right)$. Recall the decomposition $H=H_{1} \oplus H_{2}, H_{1}=\left\{v_{2 i+1}\right\}_{i}$ and $H_{2}=\left\{v_{2 i}\right\}_{i}$. Let us denote by $P_{i}, i=1,2$ as the projections to $H_{i}$. Let us put:

$$
F_{1}=i d\left[P_{2}\right] P_{2}, \quad F_{3}=i d\left[S^{*} P_{1}\right] P_{1} S .
$$

Then from the equations:

$$
P_{2} S T=P_{2} S T P_{1}=S_{35}^{*} P_{1}, \quad S^{*} P_{1} S=P_{2}
$$


this gives an extension $M_{2}\left(T, I_{0}, S\right)$ of the metabolism $S^{*}\left(M_{2}\left(T, I_{0}\right)^{\prime}\right)$. Thus one obtains a system of metabolisms $S^{*}\left(M_{2}\left(T, I_{0}\right)^{\prime}\right) \subset M_{2}\left(T, I_{0}, S\right)$.

10.E Pilbin metabolic system: There is an ensyme $P P K$ (pilbinkinase) which catalyzes from $P_{1}$ (phospho-enol-pilbin) to $P_{2}$ (pilbin). $P P K$ has two states, activating state $P P K$ and actless state $P P K^{\prime}$. These have the following system:

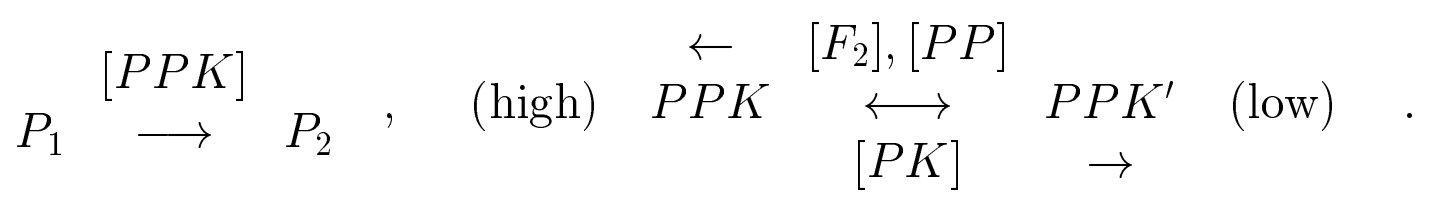

Example 10.E: Let us consider $M_{2}\left(T, I_{0}, S\right)$. Then one obtains an extension $M_{3}\left(T, I_{0}, S, S^{2}\right)$ by letting:

$$
P P K=i d\left[P_{2}\right] P_{2}, \quad P P K^{\prime}=i d\left[P_{2} T^{*}\right] T P_{2} .
$$

\section{LOOP STRUCTURE}

Let $V=W V_{1} W^{\prime} V_{2} W^{\prime \prime}$ be a m-dna sequence with a structure:

$$
\begin{aligned}
V_{1} & =a_{1}\left|X_{1}\right| a_{2} \ldots a_{l}\left|X_{l}\right| a_{l+1}, \\
V_{2} & =a_{l^{\prime}+1}\left|X_{l^{\prime}+1}\right| a_{l^{\prime}+2}|\ldots| a_{l+l^{\prime}}\left|X_{l+l^{\prime}}\right| a_{l+l^{\prime}+1} \\
& \equiv b_{l+1}\left|Y_{l}\right| b_{l}\left|\ldots b_{2}\right| Y_{1} \mid b_{1} .
\end{aligned}
$$

Suppose there exists a family of primary elements $\left\{Z_{1}, \ldots, Z_{l}\right\}$ so that the shift relation is satisfied, $a_{i} b_{i}^{*}=Z_{i}, i=1, \ldots, l$. Then the structure:

$$
\begin{array}{cccccccccc}
a_{1} & \left|X_{1}\right| & a_{2} & \left|X_{2}\right| & a_{3} & \ldots & a_{l} & \left|X_{l}\right| & a_{l+1} & \ldots \\
- & & - & & - & & - & & - & \\
Z_{1} & Z_{2} & Z_{3} & & Z_{l} & & Z_{l+1} & \\
- & - & & - & & - & & - & \\
b_{1} & \left|Y_{1}\right| & b_{2} & \left|Y_{2}\right| & b_{3} & \ldots & b_{l} & \left|Y_{l}\right| & b_{l+1} & \ldots
\end{array}
$$

is called loop structure.

11.A Parindrome: Let $V=a_{1}\left|X_{1}\right| \ldots a_{2 l-1}\left|X_{2 l-1}\right| a_{2 l}$ be a m-dna sequence 
with parindrome. Let us rewrite $V$ as $a_{1}\left|X_{1}\right| \ldots a_{l}\left|X_{l}\right| a_{l+1}\left|X_{l}\right| \ldots\left|X_{1}^{*}\right| a_{1}$. Then one obtains a loop structure by:

$$
\begin{aligned}
& \begin{array}{llllllllll}
a_{1} \mid & X_{1} & \left|a_{2}\right| & X_{2} & \left|a_{3}\right| & \ldots & \left|a_{j}\right| & X_{j} & \left|a_{j+1}\right| & \ldots
\end{array} \\
& \begin{array}{lllll}
X_{1}^{*} & X_{2}^{*} & \ldots & X_{j-1}^{*} & X_{j}^{*}
\end{array} \\
& a_{1}\left|\quad X_{1} \quad\right| a_{2}|\ldots| a_{j-1} \mid \begin{array}{llll}
X_{j-1} & \left|a_{j}\right| & \ldots
\end{array} \\
& \text { References }
\end{aligned}
$$

[ABLRRW] B.Alberts, D.Bray, J.Lewis, M.RAfF, K.Roberts And J.D.Watson, Molecular biology of the cell. 2nd ed., Garaland Publ. Inc. New York and London, (1989).

[CG] A. Carbone and M. Gromov, Mathematical slices of molecular biology, IHES preprint, (2001).

[CGP] Ed. A.Carbone, M.Gromov and P.Prusinkiewicz, Pattern formation in biology, vision and dynamics, World Scientific (2000).

[H] K.HORI, Isozyme no bunshi-seibutsu gaku, Kyouritsu-shuppan (in Japanese), 1994.

[I] Initiation la gnomique fonctionnelle: Aspects biologiques, mathmatiques et algorithmiques, seminar guide in IHES internet, www.ihes.fr.

[K] B.P.Kitchens, Symbolic dynamics, Springer, (1998).

[MM] C.L.MARKerT AND F.M $\phi$ LLER, Multiple forms of ensymes:Tissues, ontogenetic, and species patterns, Proc. Nat. Acad. Sci. USA, 45, 753-763.

[Mu] Museum National D'Histoire Naturelles, Galeries de Palentologie et Anatomie compar'ee, Jardan des plants, in Paris.

[MO] Ed. C.L.Markert and Z.Ogita, ISOZYMES: Structure, Function, and Use in Biology and Medicine, Progr. Clin.Biol.Res., Vol 344, Wiley-Liss, New York, (1990).

[U] T.UReta, The role of isozymes in metabolism: A model of metabolic pathways as the basis for the biological role of isozymes, Curr. Top. Cell. Regul., 13, 233 - 258.

\section{Tsuyoshi Kato}

Department of Mathematics

Faculty of Science

Kyoto University

Kyoto 606-01 Japan 